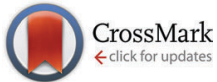

Cite this: New J. Chem., 2016, 40, 3705

Received (in Montpellier, France) 21st October 2015, Accepted 17th February 2016

DOI: $10.1039 /$ c5nj02928g

www.rsc.org/njc

\section{Surface reactivity of non-hydrolytic silicophosphate xerogels: a simple method to create Brønsted or Lewis acid sites on porous supports $\dagger$}

\author{
Ales Styskalik, ${ }^{a b}$ David Skoda, ${ }^{\text {ab }}$ Zdenek Moravec, $^{a}$ Craig E. Barnes $^{c}$ and \\ Jiri Pinkas*ab
}

\begin{abstract}
Non-hydrolytic sol-gel reactions of silicon acetates with trimethylsilyl (TMS) esters of phosphoric and phosphonic acids produce cross-linked matrices containing homogeneous dispersions of silicate and phosphoryl groups connected together by networks of $\mathrm{Si}-\mathrm{O}-\mathrm{P}(=\mathrm{O})$ linkages. The condensation degrees reach 80 to $90 \%$. Residual organic groups (10 to 20\%) were reacted with a variety of compounds $\left(\mathrm{H}_{2} \mathrm{O}, \mathrm{Me}_{3} \mathrm{SiOSiMe}_{3}, \mathrm{POCl}_{3}, \mathrm{SiCl}_{4}, \mathrm{AlMe}_{3}, \mathrm{Al}\left(\mathrm{NMe}_{2}\right)_{3}\right.$, and $\left.\mathrm{AlCl}_{3}\right)$ in order to enrich the surface of these porous matrices with Brønsted ( $\equiv \mathrm{P}-\mathrm{OH})$ and Lewis (tetracoordinated $\mathrm{Al}$ ) acid functional groups. The differences in the reactivity of $\equiv \mathrm{Si}-\mathrm{OAc}$ and $\equiv \mathrm{P}-\mathrm{OSSM}_{3}$ groups were utilized for the selective modification at the silicon and phosphorus atoms. The reaction procedures were optimized and significantly porous silicophosphate materials with a high content of either hydroxyl groups or four-coordinated aluminium species were obtained. The activity and selectivity of prepared samples as catalysts for the dimerization of $\alpha$-methylstyrene were tested. Excellent activities and moderate to very high selectivities were achieved suggesting the potential use of silicophosphate xerogels in heterogeneous catalysis.
\end{abstract}

\section{Introduction}

Chemical modification of the surfaces of inorganic supports is a common method for the fabrication of a broad spectrum of surface-engineered materials, such as chemical and biological sensors, modified electrodes, separation membranes, selective adsorbents, sorbents for HPLC, heterogeneous metal-complex catalysts, biocompatible materials, and anti-adherent or anticorrosion coatings. ${ }^{1}$ The most widely used materials are based on silica, which can be easily chemically modified with organosilicon compounds. The purpose of the post-synthesis modifications is often to introduce catalytically active groups, ${ }^{2,3}$ or to tailor the hydrophobicity of the material, ${ }^{4}$ or to change the concentration and the strength of surface acidic centers. ${ }^{5}$

Modification of the surface of silica and other oxide materials handled in air usually involves electrophilic substitution - a surface hydroxyl proton is replaced by an anchoring group of the modifier. From this it follows that the modifier molecule must

\footnotetext{
${ }^{a}$ Department of Chemistry, Masaryk University, Kotlarska 2, CZ-61137 Brno, Czech Republic. E-mail: jpinkas@chemi.muni.cz; Fax: +420549492443; Tel: +420549496493

${ }^{b}$ Masaryk University, CEITEC MU, Kamenice 5, CZ-62500 Brno, Czech Republic ${ }^{c}$ Department of Chemistry, University of Tennessee, Knoxville, TN 37996-1600, USA $\dagger$ Electronic supplementary information (ESI) available. See DOI: 10.1039/ c5nj02928g
}

contain a leaving group that forms a stable compound with the hydroxyl proton. For instance the reaction of the $\mathrm{Al}-\mathrm{Cl}$ moiety with surface $\mathrm{Si}-\mathrm{OH}$ groups has been used to anchor $\mathrm{AlCl}_{3}$ on the surface of silica (eqn (1)). ${ }^{6}$ Also $\mathrm{Me}_{3} \mathrm{SiCl}$ reacts readily with hydroxyl groups eliminating $\mathrm{HCl}$ (eqn (2)) and making the silica surface hydrophobic. ${ }^{4}$ Alkoxysilanes were applied to introduce organic functional groups (eqn (3)) allowing for a wide variety of organic reactions. As an example nitrile alkylalkoxysilane was attached to silica, the nitrile group transformed into the carboxylic group, and the final material - silica modified with carboxylic groups - was utilized for the heterogenization of metal salts. ${ }^{7}$

$$
\begin{aligned}
& \equiv \mathrm{Si}-\mathrm{OH}+\mathrm{AlCl}_{3} \rightarrow \equiv \mathrm{Si}-\mathrm{O}-\mathrm{AlCl}_{2}+\mathrm{HCl} \\
& \equiv \mathrm{Si}-\mathrm{OH}+\mathrm{Cl}-\mathrm{SiMe}_{3} \rightarrow \equiv \mathrm{Si}-\mathrm{O}-\mathrm{SiMe}_{3}+\mathrm{HCl} \\
& \equiv \mathrm{Si}-\mathrm{OH}+\mathrm{RO}-\mathrm{SiR}_{3} \rightarrow \equiv \mathrm{Si}-\mathrm{O}-\mathrm{SiR}_{3}+\mathrm{ROH}
\end{aligned}
$$

Another possibility is to chlorinate silanol groups using $\mathrm{CCl}_{4}$ or $\mathrm{SOCl}_{2}$ to increase or modify the reactivity of the silica surface. This step extends the number of possible subsequent reactions thus increasing the versatility of silica as a support for heterogeneous catalysts. ${ }^{8}$

In the case of metal and metal oxide surfaces (alumina, titania, zirconia, etc.), phosphoric and phosphonic acids are suitable grafting agents for the improvement of hydrophobic, 
anticorrosive, and other properties..$^{9-12}$ Grafting involves both the coordination of the phosphoryl oxygen to Lewis acidic metal centres, and the condensation of the $\mathrm{P}-\mathrm{OH}$ functions with surface $\mathrm{M}-\mathrm{OH}$ groups. Accordingly the coupling agent may act as a mono-, bi- or tridentate ligand on the surface. The $\mathrm{M}-\mathrm{O}-\mathrm{P}$ bonds are in most cases very stable and therefore phosphoric and phosphonic acids provide stable monolayers with high grafting densities. ${ }^{11}$ In some cases (depending on the $\mathrm{pH}$ and the metal oxide used) there are troubles with the dissolution/precipitation process which provides bulk metal phosphonates instead of metal oxides covered with a phosphonate layer. Grafting with esters of phosphonic acids in non-aqueous solvents solved this problem. ${ }^{10}$ The phosphonate layer provides better results than the siloxane layer for metal and metal oxide surfaces (stability, grafting density, etc.). Unfortunately the grafting of phosphonates onto the silica surface was shown to be problematic due to the hydrolytic instability of Si-O-P bonds. $^{13}$ However it was found recently that it proceeds smoothly with exclusion of moisture and after heating to temperatures of around $140{ }^{\circ} \mathrm{C} .{ }^{14-17}$

We recently described a new non-hydrolytic ester elimination route to synthesize amorphous microporous silicophosphate xerogels ( $\mathrm{SiP})$ by condensing silicon tetraacetate, $\mathrm{Si}(\mathrm{OAc})_{4}$, and tris(trimethylsilyl)phosphate, $\mathrm{OP}\left(\mathrm{OSiMe}_{3}\right)_{3}$ (TTP) (eqn (4)). ${ }^{18} \mathrm{Sub}$ stitution of $\mathrm{Si}(\mathrm{OAc})_{4}$ by acetoxysilanes with bridging organic groups, $(\mathrm{AcO})_{3} \mathrm{Si}-\left(\mathrm{CH}_{2}\right)_{n}-\mathrm{Si}(\mathrm{OAc})_{3}$, allowed us to synthesize completely new hybrid mesoporous silicophosphate xerogels (SiC2SiP, eqn (5)). ${ }^{19}$ Both xerogel families (parent and hybrid) are built from $\mathrm{Si}-\mathrm{O}-\mathrm{P}$ bonds, exhibit large surface areas (230 to $700 \mathrm{~m}^{2} \mathrm{~g}^{-1}$ ) and high homogeneity of Si and $\mathrm{P}$ components on the atomic scale. The degrees of condensation (DC, mol\% of eliminated trimethylsilylacetate in the course of the reaction) reach 80 to $90 \%$, and therefore xerogels possess residual organic groups, both acetoxy (coming from $\mathrm{Si}(\mathrm{OAc})_{4}$ or $(\mathrm{AcO})_{3} \mathrm{Si}-\left(\mathrm{CH}_{2}\right)_{n}{ }^{-}$ $\mathrm{Si}(\mathrm{OAc})_{3}$ precursors) and trimethylsilyloxy (coming from the $\mathrm{OP}\left(\mathrm{OSiMe}_{3}\right)_{3}$ precursor) groups. With the knowledge of the DC we can rewrite eqn (4), which describes the complete condensation of organic groups (DC $=100 \%$ ), and introduce eqn (6) for the real reaction ( $\mathrm{DC}=80 \%)$. The situation is similar in the case of hybrid xerogels with organic bridging groups.

$$
\begin{aligned}
& 3 \mathrm{Si}(\mathrm{OAc})_{4}+4 \mathrm{OP}\left(\mathrm{OSiMe}_{3}\right)_{3} \\
& \rightarrow \mathrm{Si}_{3} \mathrm{P}_{4} \mathrm{O}_{16}+12 \mathrm{CH}_{3} \mathrm{C}(\mathrm{O}) \mathrm{OSiMe}_{3}(\mathrm{DC}=100 \%) \\
&(\mathrm{AcO})_{3} \mathrm{Si}-\left(\mathrm{CH}_{2}\right)_{n}-\mathrm{Si}(\mathrm{OAc})_{3}+2 \mathrm{OP}\left(\mathrm{OSiMe}_{3}\right)_{3} \\
& \rightarrow \mathrm{OPO}_{3} \mathrm{Si}\left(\mathrm{CH}_{2}\right)_{n} \mathrm{SiO}_{3} \mathrm{PO}+ 6 \mathrm{AcOSiMe}_{3}, n=1,2,3,6 \\
& 3 \mathrm{Si}(\mathrm{OAc})_{4}+4 \mathrm{OP}\left(\mathrm{OSiMe}_{3}\right)_{3} \rightarrow \mathrm{Si}_{3} \mathrm{P}_{4} \mathrm{O}_{13.6}(\mathrm{OAc})_{2.4}\left(\mathrm{OSiMe}_{3}\right)_{2.4} \\
&+9.6 \mathrm{CH}_{3} \mathrm{C}(\mathrm{O}) \mathrm{OSiMe}_{3}(\mathrm{DC}=80 \%)
\end{aligned}
$$

Here we report on the post-synthetic chemical modification of the silicophosphate xerogel surface. The reactivities of the $\equiv \mathrm{Si}-\mathrm{OAc}$, $\equiv \mathrm{P}-\mathrm{OSiMe}_{3}$, and $\mathrm{P}=\mathrm{O}$ groups in our product, $\mathrm{Si}_{3} \mathrm{P}_{4} \mathrm{O}_{13.6}(\mathrm{OAc})_{2.4}\left(\mathrm{OSiMe}_{3}\right)_{2.4}$, are different than silanol groups on silica and allow us to use a wide variety of reactants leading to novel materials with potential use in heterogeneous catalysis.
In addition the differences in the reactivity of $\equiv \mathrm{Si}-\mathrm{OAc}$ and $\equiv \mathrm{P}-\mathrm{OSiMe}_{3}$ groups were utilized for the selective modification at the silicon and phosphorus atoms.

The work described here is divided into two parts. In the first part we focus on the detailed description of reaction pathways between residual organic groups on the surface of silicophosphate xerogels and various modifiers $\left(\mathrm{H}_{2} \mathrm{O}\right.$, hexamethyldisiloxane, $\mathrm{POCl}_{3}, \mathrm{SiCl}_{4}, \mathrm{AlMe}_{3}, \mathrm{Al}\left(\mathrm{NMe}_{2}\right)_{3}$ and $\left.\mathrm{AlCl}_{3}\right)$. The organic products of the reactions were identified by GC-MS, while the changes on the xerogel surfaces were followed mainly by IR and MAS NMR methods. The surface area of xerogels was measured by nitrogen adsorption techniques since it is the key feature of heterogeneous catalysts. The second part is devoted to the multi-step chemical modification based on the reactivity of silicophosphate xerogels, affording solid acid catalysts with either Lewis (tetracoordinated $\mathrm{Al}$ ) or Brønsted $(\equiv \mathrm{P}-\mathrm{OH})$ acidic groups. The catalytic activities of these materials are described for the dimerization of $\alpha$-methylstyrene.

\section{Experimental}

\section{General}

All manipulations were performed under dry $\mathrm{N}_{2}$ using Schlenk techniques or in an M. Braun dry box with both $\mathrm{H}_{2} \mathrm{O}$ and $\mathrm{O}_{2}$ levels below $1 \mathrm{ppm}$. $\mathrm{Si}(\mathrm{OAc})_{4}$ was prepared according to the literature. ${ }^{20} \mathrm{SiCl}_{4}$ (Merck, 99\%) and acetic acid anhydride (Riedel de Haën, 98\%) were distilled prior to use. Toluene was freshly distilled from $\mathrm{Na}$ /benzophenone under $\mathrm{N}_{2}$; pyridine, $\mathrm{CH}_{3} \mathrm{CN}, \mathrm{CH}_{2} \mathrm{Cl}_{2}$, and THF were dried by standard methods and distilled before use. Tris(trimethylsilyl)phosphate (TTP) was prepared from $\mathrm{H}_{3} \mathrm{PO}_{4}$ and $\mathrm{Me}_{3} \mathrm{SiCl}$ and vacuumdistilled. Benzene- $d_{6}$ was dried over and distilled from $\mathrm{Na} / \mathrm{K}$ alloy and degassed prior to use. $\mathrm{CDCl}_{3}$ was dried by $\mathrm{P}_{2} \mathrm{O}_{5}$ and vacuum-transferred to an ampoule. The parent silicophosphate xerogels $\mathrm{SiP}$ and $\mathrm{SiC} 2 \mathrm{SiP}$ were prepared from $\mathrm{Si}(\mathrm{OAc})_{4}$ and $(\mathrm{AcO})_{3} \mathrm{Si}-\left(\mathrm{CH}_{2}\right)_{2}-\mathrm{Si}(\mathrm{OAc})_{3}$, respectively, in reactions with $\mathrm{OP}\left(\mathrm{OSiMe}_{3}\right)_{3}$ as reported previously. ${ }^{18,19}$ Their synthesis and characterization are briefly described in the ESI. $\dagger$

\section{Characterization}

The IR spectra (4000-400 $\mathrm{cm}^{-1}$ ) were recorded on a Bruker Tensor T27 spectrometer. Samples were prepared as $\mathrm{KBr}$ pellets. Surface areas (SA) and pore volumes were determined by nitrogen adsorption at $77.4 \mathrm{~K}$ by a volumetric technique $\mathrm{e}^{21,22}$ on a Quantachrome Autosorb-1MP instrument. Prior to the measurements, the samples were degassed at $25{ }^{\circ} \mathrm{C}$ for at least $24 \mathrm{~h}$ until the outgas rate was less than $0.4 \mathrm{~Pa} \mathrm{~min}^{-1}$. The adsorption-desorption isotherm was measured for each sample at least three times. The specific surface area was determined by the multipoint BET method with at least five data points with relative pressures between 0.05 and 0.3 . Thermal analyses (TG/DSC) were performed on a Netzsch STA 449C Jupiter apparatus from 25 to $1100{ }^{\circ} \mathrm{C}$ under flowing air $\left(70 \mathrm{~cm}^{3} \mathrm{~min}^{-1}\right)$ with a heating rate of $5 \mathrm{~K} \mathrm{~min}^{-1}$. The samples for phosphorus and chlorine content estimation and alkalimetric titration with 
$0.01 \mathrm{M} \mathrm{NaOH}$ were prepared in the following way: $200 \mathrm{mg}$ of the sample was hydrolyzed in $40 \mathrm{~cm}^{3}$ of distilled water; the suspension was shaken for $24 \mathrm{~h}$. The white precipitate was filtered off and the filtrate was diluted to $250 \mathrm{~cm}^{3}$. The total amount of hydrochloric, acetic, and phosphoric acid was estimated by $\mathrm{NaOH}$ titration with a phenolphthalein indicator. The $\mathrm{Si}, \mathrm{Al}$ and $P$ contents were analyzed on an ICP OE spectrometer iCAP 6500 Duo (Thermo, generator 27.12 MHz, amplitude $1.15 \mathrm{~kW}$, plasma gas flow $12 \mathrm{dm}^{3} \mathrm{~min}^{-1}$ ) using emission lines 212.412 and $251.611 \mathrm{~nm}$ for $\mathrm{Si}, 167.079$ and $308.215 \mathrm{~nm}$ for $\mathrm{Al}$, and 213.618 and $214.914 \mathrm{~nm}$ for $\mathrm{P}$. The chlorine content was analyzed by argentometric titration. Solution NMR spectra were recorded on a Bruker Avance II 300 NMR spectrometer at frequencies $300.1 \mathrm{MHz}$ for ${ }^{1} \mathrm{H}$ and $75.5 \mathrm{MHz}$ for ${ }^{13} \mathrm{C}$ with deuterated solvents as the external lock. The ${ }^{1} \mathrm{H}$ and ${ }^{13} \mathrm{C}\left\{{ }^{1} \mathrm{H}\right\}$ NMR spectra were referenced to the residual proton signals or carbon resonances of benzene- $d_{6}$ (7.15 and $128.0 \mathrm{ppm}$, respectively) and $\mathrm{CDCl}_{3}$ (7.26 and $\left.77.0 \mathrm{ppm}\right)$. Solid-state ${ }^{27} \mathrm{Al}$ and ${ }^{31} \mathrm{P}$ MAS, and ${ }^{29} \mathrm{Si}$ and ${ }^{13} \mathrm{C}$ CPMAS NMR spectra were acquired on a wide-bore Varian INOVA 400 NMR spectrometer with a broadband Chemagnetic $5 \mathrm{~mm}$ MAS probe. Inside a dry box, samples were loaded into $5 \mathrm{~mm}$ pencil rotors, stoppered with teflon plugs and sealed with silicon grease and paraffin wax. Magic angle spinning rates were $5 \mathrm{kHz}$ for ${ }^{29} \mathrm{Si}$ and ${ }^{13} \mathrm{C}$ CPMAS and $10 \mathrm{kHz}$ for ${ }^{31} \mathrm{P}$ and ${ }^{27} \mathrm{Al}$ MAS spectra. Chemical shifts were referenced externally to ${ }^{31} \mathrm{P} \delta\left[\mathrm{H}_{3} \mathrm{PO}_{4}(85 \%)\right]: 0.0 \mathrm{ppm} ;{ }^{29} \mathrm{Si} \delta$ $\left[\left(\mathrm{Me}_{3} \mathrm{SiO}\right)_{8} \mathrm{Si}_{8} \mathrm{O}_{20}\right]: 11.72 \mathrm{ppm} ;{ }^{13} \mathrm{C} \delta$ [adamantane]: $38.68 \mathrm{ppm}$; ${ }^{27} \mathrm{Al} \delta\left[\left[\mathrm{Al}\left(\mathrm{H}_{2} \mathrm{O}\right)_{6}\right]^{3+}\right.$ (aq. solution)]: $0.0 \mathrm{ppm}$. GC-MS spectra were obtained on a Thermo Scientific Trace GC Ultra - TSQ Quantum XLS mass spectrometer. A TS-SQC column $(15 \mathrm{~m}, 0.25 \mathrm{~mm}$, $0.25 \mu \mathrm{m})$ was heated with the following program: $50{ }^{\circ} \mathrm{C}(0 \mathrm{~min})$, $5{ }^{\circ} \mathrm{C} \mathrm{min}{ }^{-1}$ up to $80{ }^{\circ} \mathrm{C}, 15{ }^{\circ} \mathrm{C} \min ^{-1}$ up to $120{ }^{\circ} \mathrm{C}$, and $35{ }^{\circ} \mathrm{C}$ $\min ^{-1}$ up to $200{ }^{\circ} \mathrm{C}(0.5 \mathrm{~min})$; split mode, injector temperature $200{ }^{\circ} \mathrm{C}$, interface temperature $200{ }^{\circ} \mathrm{C}$, and detector temperature $200{ }^{\circ} \mathrm{C}$. The column pressure was set to $31.5 \mathrm{kPa}$ and ionization energy to $70 \mathrm{eV}$.

\section{Chemical modification of silicophosphate xerogel surfaces}

\section{Modification with $\mathrm{H}_{2} \mathrm{O}$}

A suspension of $\mathrm{SiP}\left(0.814 \mathrm{~g}, 2.52 \mathrm{mmol}\right.$ of residual AcOSiMe $\mathrm{A}_{3}$, i.e. xerogel contained $2.52 \mathrm{mmol} \mathrm{AcO}-\mathrm{Si} \equiv$ and $2.52 \mathrm{mmol}$ $\mathrm{Me}_{3} \mathrm{SiO}-\mathrm{P} \equiv$ groups) in $40.7 \mathrm{~cm}^{3}$ of $\mathrm{THF}$ was reacted with $\mathrm{H}_{2} \mathrm{O}(0.0433 \mathrm{~g}, 2.40 \mathrm{mmol}, 50 \%$ relative to residual groups) at r.t. for $24 \mathrm{~h}$. The resulting xerogel ( $\mathrm{SiPH} 2)$ was isolated by drying for $48 \mathrm{~h}$ at r.t. in vacuo. The reaction was performed similarly with the $\mathrm{H}_{2} \mathrm{O}$-to-residual group ratio of 100 and $5 \%$ (Table 1).

Characterization and analyses of the SiPH2 xerogel.

IR (SiPH2, KBr, cm ${ }^{-1}$ ) $\nu: 505 \mathrm{~m}, 606 \mathrm{vw}, 689 \mathrm{vw}, 762 \mathrm{~m}$ $\left(\rho_{\mathrm{s}} \mathrm{SiCH}_{3}\right), 852$ vs $\left(\rho_{\text {as }} \mathrm{SiCH}_{3}\right), 1074$ vs $(\nu \mathrm{Si}-\mathrm{O}-\mathrm{P}), 1095$ vs $(\nu \mathrm{P}-\mathrm{O}-\mathrm{Si}), 1259 \mathrm{~s}\left(\delta_{\mathrm{s}} \mathrm{SiCH}_{3}\right), 1424 \mathrm{w}\left(\delta_{\mathrm{as}} \mathrm{CH}_{3}\right), 1662 \mathrm{w}$ $(\delta \mathrm{OH}), 2908 \mathrm{vw}\left(\nu_{\mathrm{s}} \mathrm{CH}_{3}\right), 2965 \mathrm{w}\left(\nu_{\mathrm{as}} \mathrm{CH}_{3}\right)$.

${ }^{13} \mathrm{C}$ CPMAS NMR (SiPH2, ppm) $\delta:-3.6\left(\mathrm{POSiCH}_{3}\right)$.
Table 1 Chemical modification of xerogels with water. Molar amounts of residual organic groups and water used in the reactions. Relative amounts of water

\begin{tabular}{llllll}
\hline Sample & $\begin{array}{l}m_{\text {xerogel }} \\
{[\mathrm{g}]}\end{array}$ & $\begin{array}{l}n_{\mathrm{AcOSiMe}_{3}} \\
{[\mathrm{mmol}]}\end{array}$ & $\begin{array}{l}n_{\mathrm{H}_{2} \mathrm{O}} \\
{[\mathrm{mmol}]}\end{array}$ & $\begin{array}{l}n_{\mathrm{H}_{2} \mathrm{O}}{ }^{2} \\
{[\%]}\end{array}$ & $\begin{array}{l}\text { Yield } \\
{[\mathrm{g}]}\end{array}$ \\
\hline SiPH1 & 0.570 & 1.77 & 0.17 & 5 & - \\
$\mathrm{SiPH} 2$ & 0.814 & 2.52 & 2.40 & 50 & - \\
$\mathrm{SiPH} 3$ & 0.946 & 2.93 & 5.58 & 100 & 0.967 \\
$\mathrm{SiC} 2 \mathrm{SiPH} 1$ & 0.234 & 0.42 & 0.72 & 100 & 0.202 \\
${ }^{a}$ Relative to residual AcO-Si $\equiv$ and $\mathrm{Me}_{3} \mathrm{SiO}-\mathrm{P} \equiv$ organic groups.
\end{tabular}

${ }^{29} \mathrm{Si}$ CPMAS NMR (SiPH2, ppm) $\delta: 23.6\left(\mathrm{POSiMe}_{3}\right),-115$ $\left(\mathrm{SiO}_{4}\right),-214\left(\mathrm{SiO}_{6}\right)$.

${ }^{31} \mathrm{P}$ MAS NMR (SiPH2, ppm) $\delta:-47 \quad\left(\mathrm{P}(\mathrm{OSi})_{4}\right), \quad-36$ $\left(\mathrm{P}(\mathrm{OH})(\mathrm{OSi})_{3}\right), \quad-26 \quad\left(\mathrm{P}(\mathrm{OH})_{2}(\mathrm{OSi})_{2}\right), \quad-16 \quad\left(\mathrm{P}(\mathrm{OH})_{3}(\mathrm{OSi}), \quad-8\right.$ $\left(\mathrm{O}=\mathrm{P}(\mathrm{OH})_{2}(\mathrm{OSi})\right)$.

TG/DSC (air, $5 \mathrm{~K} \mathrm{~min}^{-1}$ ): weight loss at $1000{ }^{\circ} \mathrm{C}: 40.6 \%$.

BET: nonporous

Data for SiPH1, SiPH3 and SiC2SiPH1 see ESI. $\dagger$

\section{Modification with hexamethyldisiloxane}

$\mathrm{Me}_{3} \mathrm{SiOSiMe}_{3}$ (0.7146 g, $4.401 \mathrm{mmol}$ ) was added dropwise through a syringe to the suspension of $\operatorname{SiP}(1.820 \mathrm{~g}, 4.680 \mathrm{mmol}$ of residual $\mathrm{AcOSiMe}_{3}$ ) in $15 \mathrm{~cm}^{3}$ of toluene. The reaction mixture was kept at $80{ }^{\circ} \mathrm{C}$ for one week. Volatile organic products were identified by GC-MS (trimethylsilylacetate, $\mathrm{m} / \mathrm{z}$ 45/61/75/117) and the inorganic product (SiPHMDSO, $2.013 \mathrm{~g}$ ) was isolated by drying for $48 \mathrm{~h}$ at r.t. in vacuo. The same reaction was performed with $\mathrm{Me}_{3} \mathrm{SiOSiMe}_{3}(0.5241 \mathrm{~g}, 3.228 \mathrm{mmol})$ and hybrid SiC2SiP xerogel $(1.450 \mathrm{~g}, 2.970 \mathrm{mmol}$ of residual $\mathrm{AcOSiMe}_{3}$ ) affording $0.9128 \mathrm{~g}$ of white xerogel (SiC2SiPHMDSO).

Characterization and analyses of the SiPHMDSO xerogel.

TG/DSC (air, $5 \mathrm{~K} \mathrm{~min}^{-1}$ ): weight loss at $1000{ }^{\circ} \mathrm{C}$ : $53.5 \%$.

IR (SiPHMDSO, KBr, cm $\left.{ }^{-1}\right) \nu: 502 \mathrm{~m}, 606 \mathrm{vw}, 654 \mathrm{vw}\left(\nu \mathrm{SiC}_{3}\right)$, $692 \mathrm{vw}, 762 \mathrm{~m}\left(\rho_{\mathrm{s}} \mathrm{SiCH}_{3}\right), 849 \mathrm{vs}\left(\rho_{\mathrm{as}} \mathrm{SiCH}_{3}\right), 1035 \mathrm{~s}(\nu \mathrm{Si}-\mathrm{O}-\mathrm{P})$, $1100 \mathrm{~s}(\nu \mathrm{P}-\mathrm{O}-\mathrm{Si}), 1256 \mathrm{~s}\left(\delta_{\mathrm{s}} \mathrm{SiCH}_{3}\right) 1300 \mathrm{sh}(\nu \mathrm{P}=\mathrm{O}), 1418 \mathrm{w}$ $\left(\delta_{\text {as }} \mathrm{CH}_{3}\right), 1541 \mathrm{vw}\left(\nu_{\mathrm{s}} \mathrm{COO}\right.$ bidentate $), 2904 \mathrm{w}\left(\nu_{\mathrm{s}} \mathrm{CH}_{3}\right), 2962 \mathrm{~m}$ $\left(\nu_{\text {as }} \mathrm{CH}_{3}\right)$.

${ }^{13} \mathrm{C}$ CPMAS NMR (SiPHMDSO, ppm) $\delta: 3.8\left(\mathrm{POSiCH}_{3}+\right.$ $\mathrm{SiOSiCH}_{3}$ ).

${ }^{29} \mathrm{Si}$ CPMAS NMR (SiPHMDSO, ppm) $\delta: 21.4\left(\mathrm{POSiMe}_{3}\right), 13.6$ $\left(\mathrm{SiOSiMe}_{3}\right),-111\left(\mathrm{SiO}_{4}\right),-214\left(\mathrm{SiO}_{6}\right)$.

${ }^{31} \mathrm{P}$ MAS NMR (SiPHMDSO, ppm) $\delta:-45\left(\mathrm{P}(\mathrm{OSi})_{4}\right),-29$ $\left(\mathrm{O}=\mathrm{P}(\mathrm{OSi})_{3}\right)$.

BET: $107 \mathrm{~m}^{2} \mathrm{~g}^{-1}$.

Data for SiC2SiPHMDSO are provided in the ESI. $\dagger$

\section{Modification with silicon tetrachloride}

$\mathrm{SiCl}_{4}(0.261 \mathrm{~g}, 1.53 \mathrm{mmol})$ was vacuum transferred to the suspension of SiP $\left(1.034 \mathrm{~g}, 3.26 \mathrm{mmol}\right.$ of residual $\mathrm{AcOSiMe}_{3}$, $1 \mathrm{Cl}: 1$-OAc or $-\mathrm{OSiMe}_{3}$ group) in $10 \mathrm{~cm}^{3}$ of toluene. The reaction temperature of $80{ }^{\circ} \mathrm{C}$ was maintained for $24 \mathrm{~h}$. After that, the solvent and volatile products (GC-MS: $\mathrm{CH}_{3} \mathrm{COCl}, \mathrm{m} / \mathrm{z}$ 43/63/65, $\mathrm{Me}_{3} \mathrm{SiCl}, \mathrm{m} / \mathrm{z} 73 / 93 / 95 / 108$ ) were removed in vacuo affording white xerogel, which was dried for $48 \mathrm{~h}$ at r.t. in vacuo (SiPSi, $0.913 \mathrm{~g}$ ). The same reaction was performed with $\mathrm{PhCH}_{2} \mathrm{SiCl}_{3}$ 
(0.228 g, $1.02 \mathrm{mmol})$ and SiP xerogel $(1.003 \mathrm{~g}, 1.495 \mathrm{mmol}$ of residual $\mathrm{AcOSiMe}_{3}, 1 \mathrm{Cl}: 1$-OAc or $-\mathrm{OSiMe}_{3}$ group) affording $1.036 \mathrm{~g}$ of white xerogel $\left(\mathrm{SiPPhCH}_{2} \mathrm{Si}\right)$.

Characterization and analyses of the SiPSi xerogel.

TG/DSC (air, $5 \mathrm{~K} \mathrm{~min}^{-1}$ ): weight loss at $1000{ }^{\circ} \mathrm{C}: 24.6 \%$.

IR (SiPSi, KBr, cm $\left.{ }^{-1}\right) \nu: 496 \mathrm{~m}, 646 \mathrm{w}, 764 \mathrm{w}\left(\rho_{\mathrm{s}} \mathrm{SiCH}_{3}\right)$, $853 \mathrm{~m}\left(\rho_{\text {as }} \mathrm{SiCH}_{3}\right), 1022$ vs $\left(\rho \mathrm{CH}_{3}\right), 1049$ vs $(\nu \mathrm{Si}-\mathrm{O}-\mathrm{P}), 1113$ vs $(\nu \mathrm{P}-\mathrm{O}-\mathrm{Si}), 1261 \mathrm{w}\left(\delta_{\mathrm{s}} \mathrm{SiCH}_{3}\right) 1316 \mathrm{sh}(\nu \mathrm{P}=\mathrm{O}), 1375 \mathrm{w}\left(\delta_{\mathrm{s}} \mathrm{CH}_{3}\right)$, $1418 \mathrm{vw}\left(\delta_{\mathrm{as}} \mathrm{CH}_{3}\right), 1541 \mathrm{~m}\left(\nu_{\mathrm{s}} \mathrm{COO}\right), 1724 \mathrm{vw}\left(\nu_{\mathrm{as}} \mathrm{COO}\right), 1773 \mathrm{~m}$ ( $\left.\nu_{\text {as }} \mathrm{COO}\right), 2912 \mathrm{vw}\left(\nu_{\mathrm{s}} \mathrm{CH}_{3}\right), 2964 \mathrm{w}\left(\nu_{\mathrm{as}} \mathrm{CH}_{3}\right)$.

${ }^{13} \mathrm{C}$ CPMAS NMR (SiPSi, ppm) $\delta: 1.8\left(\mathrm{POSiCH}_{3}\right), 18.4$ ( $\mathrm{CH}_{3} \mathrm{COO}$ bidentate), $23.6\left(\mathrm{CH}_{3} \mathrm{COO}\right.$ unidentate $), 169.1\left(\mathrm{CH}_{3} \mathrm{COO}\right.$ unidentate).

${ }^{29} \mathrm{Si}$ CPMAS NMR (SiPSi, ppm) $\delta: 29.0\left(\mathrm{POSiMe}_{3}\right),-67.8$ $\left(\mathrm{Cl}_{2} \mathrm{SiO}_{2}\right),-91.8\left(\mathrm{ClSiO}_{3}\right),-111\left(\mathrm{SiO}_{4}\right),-196\left(\mathrm{SiO}_{6}\right),-215\left(\mathrm{SiO}_{6}\right)$.

${ }^{31} \mathrm{P}$ MAS NMR (SiPSi, ppm) $\delta:-42\left(\mathrm{P}(\mathrm{OSi})_{4}\right)$.

BET: $865 \mathrm{~m}^{2} \mathrm{~g}^{-1}$.

Data for $\mathrm{SiPPhCH}_{2} \mathrm{Si}$ are provided in the ESI. $\dagger$

\section{Modification with phosphoryl trichloride}

$\mathrm{POCl}_{3}(0.302 \mathrm{~g}, 1.97 \mathrm{mmol})$ was added dropwise through a syringe to the suspension of $\mathrm{SiP}(1.050 \mathrm{~g}, 3.30 \mathrm{mmol}$ of residual $\mathrm{AcOSiMe}_{3}$ ) in $10 \mathrm{~cm}^{3}$ of toluene. The reaction mixture was kept at $80{ }^{\circ} \mathrm{C}$ for one week. Volatile products were identified by GC-MS $\left(\mathrm{CH}_{3} \mathrm{COCl}, m / z\right.$ 43/63/65, $\mathrm{Me}_{3} \mathrm{SiCl}, \mathrm{m} / z$ 73/93/95/108, $\left.\mathrm{POCl}_{3}, \mathrm{~m} / \mathrm{z} 47 / 82 / 117 / 119 / 152 / 156\right)$ and the inorganic product (SiPP, $1.099 \mathrm{~g}$ ) was isolated after drying for $48 \mathrm{~h}$ at r.t. in vacuo.

Characterization and analyses of the SiPP xerogel.

TG/DSC (air, $5 \mathrm{~K} \mathrm{~min}^{-1}$ ): weight loss at $1000{ }^{\circ} \mathrm{C}: 21.8 \%$.

IR (SiPP, KBr, cm $\left.{ }^{-1}\right) \nu: 489 \mathrm{~m}, 759 \mathrm{w}\left(\rho_{\mathrm{s}} \mathrm{SiCH}_{3}\right), 853 \mathrm{~m}$ $\left(\rho_{\text {as }} \mathrm{SiCH}_{3}\right), 1049$ vs $(\nu \mathrm{Si}-\mathrm{O}-\mathrm{P}), 1093$ vs $(\nu \mathrm{P}-\mathrm{O}-\mathrm{Si}), 1261 \mathrm{~W}$ $\left(\delta_{\mathrm{s}} \mathrm{SiCH}_{3}\right) 1302 \mathrm{sh}(\nu \mathrm{P}=\mathrm{O}), 2918 \mathrm{vw}\left(\nu_{\mathrm{s}} \mathrm{CH}_{3}\right), 2965 \mathrm{w}\left(\nu_{\mathrm{as}} \mathrm{CH}_{3}\right)$.

${ }^{13} \mathrm{C}$ CPMAS NMR (SiPP, ppm) $\delta: 2.8 \quad\left(\mathrm{POSiCH}_{3}\right), 24.5$ $\left(\mathrm{CH}_{3} \mathrm{COO}\right.$ unidentate $)$.

${ }^{29} \mathrm{Si} \mathrm{CPMAS} \mathrm{NMR} \mathrm{(SiPP,} \mathrm{ppm)} \delta: 26.8\left(\mathrm{POSiMe}_{3}\right),-108\left(\mathrm{SiO}_{4}\right)$, $-214\left(\mathrm{SiO}_{6}\right)$.

${ }^{31} \mathrm{P}$ MAS NMR (SiPP, ppm) $\delta:-40\left(\mathrm{P}(\mathrm{OSi})_{4}\right),-31\left(\mathrm{ClP}(\mathrm{OSi})_{3} /\right.$ $\left.\mathrm{O}=\mathrm{P}(\mathrm{OSi})_{3}\right)$.

BET: $483 \mathrm{~m}^{2} \mathrm{~g}^{-1}$.

\section{Modification with trimethylaluminium}

$\mathrm{AlMe}_{3}(0.170 \mathrm{~g}, 1.18 \mathrm{mmol})$ was reacted with xerogel SiP $\left(1.010 \mathrm{~g}, 3.35 \mathrm{mmol}\right.$ of residual $\left.\mathrm{AcOSiMe}_{3}\right)$ in toluene $\left(10 \mathrm{~cm}^{3}\right)$ at r.t. for $18 \mathrm{~h}$. GC-MS of volatile organic products: tetramethylsilane $\mathrm{m} / \mathrm{z} 73 / 45$, acetone $\mathrm{m} / \mathrm{z} 58 / 43$, hexamethyldisiloxane $\mathrm{m} / \mathrm{z}$ $147 / 131 / 73$. The resulting xerogel SiPAl1 (1.019 g) was dried in vacuo for $48 \mathrm{~h}$.

Characterization and analyses of the SiPAl1 xerogel.

TG/DSC (air, $5 \mathrm{~K} \mathrm{~min}^{-1}$ ): weight loss at $1000{ }^{\circ} \mathrm{C}: 26.2 \%$.

IR (SiPAl1, KBr, cm $\left.{ }^{-1}\right) \nu: 489 \mathrm{~m}, 689 \mathrm{~m}\left(\nu_{\mathrm{as}} \mathrm{AlC}_{3}\right), 766 \mathrm{w}$ $\left(\rho_{\mathrm{s}} \mathrm{SiCH}_{3}\right), 856 \mathrm{~s}\left(\rho_{\text {as }} \mathrm{SiCH}_{3}\right), 1024 \mathrm{~s}\left(\rho \mathrm{CH}_{3}\right), 1052 \mathrm{vs}(\nu \mathrm{Si}-\mathrm{O}-\mathrm{P})$, 1116 vs $(\nu \mathrm{P}-\mathrm{O}-\mathrm{Si}), 1193 \mathrm{vs}\left(\delta_{\mathrm{s}} \mathrm{AlCH}_{3}\right), 1260 \mathrm{~s}\left(\delta_{\mathrm{s}} \mathrm{SiCH}_{3}\right) 1311 \mathrm{sh}$ $(\nu \mathrm{P}=\mathrm{O}), 1373 \mathrm{vw}\left(\delta_{\mathrm{s}} \mathrm{CH}_{3}\right), 1420 \mathrm{vw}\left(\delta_{\mathrm{as}} \mathrm{CH}_{3}\right), 1477 \mathrm{w}\left(\nu_{\mathrm{s}} \mathrm{COO}\right)$, $1547 \mathrm{w}\left(\nu_{\mathrm{s}} \mathrm{COO}\right), 1604 \mathrm{w}\left(\nu_{\mathrm{as}} \mathrm{COO}\right), 1771 \mathrm{~m}\left(\nu_{\mathrm{as}} \mathrm{C}=\mathrm{O}\right), 2918 \mathrm{vw}$ $\left(\nu_{\mathrm{s}} \mathrm{CH}_{3}\right), 2937 \mathrm{~m}\left(\nu_{\text {as }} \mathrm{AlCH}_{3}\right), 2965 \mathrm{w}\left(\nu_{\text {as }} \mathrm{CH}_{3}\right)$.
${ }^{13} \mathrm{C}$ CPMAS NMR (SiPAl1, ppm) $\delta:-7.9\left(\mathrm{AlCH}_{3}\right), 1.8$ $\left(\mathrm{POSiCH}_{3}\right), 32.4\left(\mathrm{CH}_{3} \mathrm{COO}\right)$.

${ }^{29} \mathrm{Si}$ CPMAS NMR (SiPAl1, ppm) $\delta: 24.4\left(\mathrm{POSiMe}_{3}\right), 16.6$ $\left(\mathrm{SiOSiMe}_{3}\right),-3.7\left(\mathrm{Me}_{2} \mathrm{SiO}_{2}\right),-64\left(\mathrm{MeSiO}_{3}\right),-115\left(\mathrm{SiO}_{4}\right),-214$ $\left(\mathrm{SiO}_{6}\right)$.

${ }^{31} \mathrm{P}$ MAS NMR (SiPAl1, ppm) $\delta:-39\left(\mathrm{O}=\mathrm{P}(\mathrm{OSi} / \mathrm{Al})_{3}\right)$.

${ }^{27} \mathrm{Al}$ MAS NMR (SiPAl1, ppm) $\delta:-7\left({ }^{[6]} \mathrm{Al}\right.$, hexacoordinated $\mathrm{Al}$ atom).

BET: nonporous.

\section{Modification with tris(dimethylamido)aluminum}

Dry toluene $\left(10 \mathrm{~cm}^{3}\right)$ was poured on the mixture of solid $\mathrm{Al}\left(\mathrm{NMe}_{2}\right)_{3}(0.722 \mathrm{~g}, 4.53 \mathrm{mmol})$ and solid xerogel SiP (2.018 g, $6.85 \mathrm{mmol}$ of residual $\mathrm{AcOSiMe}_{3}$ ). The reaction mixture reacted for $18 \mathrm{~h}$ at r.t. After drying in vacuo for $48 \mathrm{~h}$ at r.t., $2.534 \mathrm{~g}$ of yellowish xerogel (SiPAl2) was obtained. GC-MS of volatile organic products: dimethyl(trimethylsilyl)amine $\mathrm{m} / \mathrm{z}$ 117/102/ $73 / 59$, dimethylacetamide $m / z 87 / 72 / 44$, hexamethyldisiloxane $m / z 147 / 131 / 73$.

Characterization and analyses of the SiPAl 2 xerogel.

TG/DSC (air, $5 \mathrm{~K} \mathrm{~min}^{-1}$ ): weight loss at $1000{ }^{\circ} \mathrm{C}: 38.8 \%$.

IR (SiPAl2, KBr, cm $\left.{ }^{-1}\right) \nu: 495 \mathrm{~m}, 758 \mathrm{w}\left(\rho_{\mathrm{s}} \mathrm{SiCH}_{3}\right), 852 \mathrm{vs}$ $\left(\rho_{\text {as }} \mathrm{SiCH}_{3}\right), 1006 \mathrm{~s}\left(\rho \mathrm{CH}_{3}\right), 1052$ vs $(\nu \mathrm{Si}-\mathrm{O}-\mathrm{P}), 1099$ vs $(\nu \mathrm{P}-\mathrm{O}-$ $\mathrm{Si}), 1257 \mathrm{~s}\left(\delta_{\mathrm{s}} \mathrm{SiCH}_{3}\right) 1311 \mathrm{sh}(\nu \mathrm{P}=\mathrm{O}), 1373 \mathrm{vw}\left(\delta_{\mathrm{s}} \mathrm{CH}_{3}\right), 1410$ vw ( $\left.\delta_{\text {as }} \mathrm{CH}_{3}\right), 1467 \mathrm{w}\left(\nu_{\mathrm{s}} \mathrm{COO}\right), 1627 \mathrm{w}$ ( $\left.\nu_{\text {as }} \mathrm{COO}\right), 2809 \mathrm{w}, 2903$ vw $\left(\nu_{\mathrm{s}} \mathrm{CH}_{3}\right), 2960 \mathrm{w}\left(\nu_{\mathrm{as}} \mathrm{CH}_{3}\right)$.

${ }^{13} \mathrm{C}$ CPMAS NMR (SiPAl2, ppm) $\delta:-2.9\left(\mathrm{POSiCH}_{3}\right), 14.4$ ( $\mathrm{CH}_{3} \mathrm{COO}$ bident), $16.9\left(\mathrm{CH}_{3} \mathrm{COO}\right.$ bident), $20.1\left(\mathrm{CH}_{3} \mathrm{COO}\right.$ unident), $32.7\left(\mathrm{~N}\left(\mathrm{CH}_{3}\right)_{2}\right), 39.0\left(\mathrm{~N}\left(\mathrm{CH}_{3}\right)_{2}\right), 166\left(\mathrm{CH}_{3} \mathrm{COO}\right.$ unident $)$, $174\left(\mathrm{CH}_{3} \mathrm{COO}\right.$ bident).

${ }^{29} \mathrm{Si}$ CPMAS NMR (SiPAl2, ppm) $\delta: 17.7$ (POSiMe $\left.{ }_{3}\right), 0.4$ $\left(\mathrm{AlOSiMe}_{3}\right),-68.5\left(\left(\mathrm{Me}_{2} \mathrm{~N}\right)_{2} \mathrm{SiO}_{2}\right),-90\left(\mathrm{SiO}_{4}+\mathrm{Me}_{2} \mathrm{NSiO}_{3}\right)$, $-110\left(\mathrm{SiO}_{4}\right),-206\left(\mathrm{SiO}_{6}\right),-214\left(\mathrm{SiO}_{6}\right)$.

${ }^{31} \mathrm{P}$ MAS NMR (SiPAl2, ppm) $\delta:-42\left(\mathrm{P}(\mathrm{OSi})_{4}\right)$.

${ }^{27} \mathrm{Al}$ MAS NMR (SiPAl2, ppm) $\delta: 35\left({ }^{[5]} \mathrm{Al}\right),-17\left({ }^{[6]} \mathrm{Al}\right)$. BET: $375 \mathrm{~m}^{2} \mathrm{~g}^{-1}$.

\section{Modification with aluminum trichloride}

Dry toluene $\left(10 \mathrm{~cm}^{3}\right)$ was poured on a mixture of solid $\mathrm{AlCl}_{3}$ $(0.204 \mathrm{~g}, 1.53 \mathrm{mmol})$ and solid xerogel SiP (0.585 g, $1.99 \mathrm{mmol}$ of residual $\mathrm{AcOSiMe}_{3}$ ). The reaction mixture reacted for $18 \mathrm{~h}$ at r.t. After drying in vacuo for $48 \mathrm{~h}$ at r.t., $0.647 \mathrm{~g}$ of white xerogel (SiPAl3) was obtained. GC-MS of volatile organic products: trimethylchlorosilane $\mathrm{m} / \mathrm{z}$ 95/93/73, hexamethyldisiloxane $\mathrm{m} / \mathrm{z}$ 147/131/73.

Characterization and analyses of the SiPAl3 xerogel.

TG/DSC (air, $5 \mathrm{~K} \mathrm{~min}^{-1}$ ): weight loss at $1000{ }^{\circ} \mathrm{C}: 31.7 \%$.

IR (SiPAl3, KBr, cm $\left.{ }^{-1}\right) \nu: 502 \mathrm{~m}, 652 \mathrm{vw}, 762 \mathrm{w}\left(\rho_{\mathrm{s}} \mathrm{SiCH}_{3}\right)$, $855 \mathrm{~m}\left(\rho_{\text {as }} \mathrm{SiCH}_{3}\right), 1051 \mathrm{~s}(\nu \mathrm{Si}-\mathrm{O}-\mathrm{P}), 1148 \mathrm{vs}(\nu \mathrm{P}-\mathrm{O}-\mathrm{Si}), 1262 \mathrm{~s}$ $\left(\delta_{\mathrm{s}} \mathrm{SiCH}_{3}\right) 1304 \mathrm{sh}(\nu \mathrm{P}=\mathrm{O}), 1378 \mathrm{vw}\left(\delta_{\mathrm{s}} \mathrm{CH}_{3}\right), 1418 \mathrm{vw}\left(\delta_{\mathrm{as}} \mathrm{CH}_{3}\right)$, $1482 \mathrm{w}\left(\nu_{\mathrm{s}} \mathrm{COO}\right), 1545 \mathrm{w}\left(\nu_{\mathrm{s}} \mathrm{COO}\right), 1602 \mathrm{~m}$ ( $\left.\nu_{\mathrm{as}} \mathrm{COO}\right), 1772 \mathrm{w}$ ( $\left.\nu_{\text {as }} \mathrm{COO}\right), 2908 \mathrm{vw}\left(\nu_{\mathrm{s}} \mathrm{CH}_{3}\right), 2967 \mathrm{w}\left(\nu_{\text {as }} \mathrm{CH}_{3}\right)$.

${ }^{13} \mathrm{C}$ CPMAS NMR (SiPAl3, ppm) $\delta: 1.1\left(\mathrm{POSiCH}_{3}\right), 18.5$ ( $\mathrm{CH}_{3} \mathrm{COO}$ bident), $22.9\left(\mathrm{CH}_{3} \mathrm{COO}\right.$ unident), $168.1\left(\mathrm{CH}_{3} \mathrm{COO}\right.$ unident), 181.6 ( $\mathrm{CH}_{3} \mathrm{COO}$ bident), 194.3 ( $\mathrm{CH}_{3} \mathrm{COO}$ bident). 
${ }^{29} \mathrm{Si}$ CPMAS NMR (SiPAl3, ppm) $\delta: 29.1\left(\mathrm{POSiMe}_{3}\right),-91$ $\left(\mathrm{ClSiO}_{3}\right),-113\left(\mathrm{SiO}_{4}\right),-196\left(\mathrm{SiO}_{6}\right),-214\left(\mathrm{SiO}_{6}\right)$.

${ }^{31} \mathrm{P}$ MAS NMR (SiPAl3, ppm) $\delta:-49\left(\mathrm{P}(\mathrm{OSi} / \mathrm{Al})_{4}\right)$.

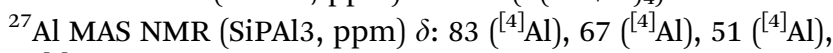
$-23\left({ }^{[6]} \mathrm{Al}\right)$.

BET: $469 \mathrm{~m}^{2} \mathrm{~g}^{-1}$.

\section{Preparation of solid acid catalysts}

Lewis acidic catalysts (SiPL and SiC2SiPL). The silicophosphate xerogels were reacted with hexamethyldisiloxane $\left(\mathrm{Me}_{3} \mathrm{SiOSiMe}_{3}\right.$ : residual organic groups ratio $\left.=1: 2\right)$ in order to deplete acetoxy groups by elimination of $\mathrm{Me}_{3} \mathrm{SiOAc}$. Subsequent reaction with $\mathrm{AlCl}_{3}(\mathrm{Cl}$ : residual organic group ratio = $1: 1)$ resulted in the elimination of $\mathrm{Me}_{3} \mathrm{SiCl}$. Reactions were carried out in toluene at temperatures and for times described in Part 1: Chemical modification of silicophosphate xerogel surfaces.

IR (SiPL, KBr, cm ${ }^{-1}$ ) $\nu: 525 \mathrm{~m}, 606 \mathrm{vw}, 692 \mathrm{w}, 763 \mathrm{~m}$ $\left(\rho_{\mathrm{s}} \mathrm{SiCH}_{3}\right), 853$ vs $\left(\rho_{\mathrm{as}} \mathrm{SiCH}_{3}\right), 1096$ vs $(\nu \mathrm{Si}-\mathrm{O}-\mathrm{P}), 1186 \mathrm{~s}$ $(\nu \mathrm{P}-\mathrm{O}-\mathrm{Si}), 1258 \mathrm{~s}\left(\delta_{\mathrm{s}} \mathrm{SiCH}_{3}\right), 1419 \mathrm{vw}\left(\delta_{\mathrm{as}} \mathrm{CH}_{3}\right), 1462 \mathrm{vw}$, $2909 \mathrm{vw}\left(\nu_{\mathrm{s}} \mathrm{CH}_{3}\right), 2966 \mathrm{~m}\left(\nu_{\mathrm{as}} \mathrm{CH}_{3}\right)$.

${ }^{13} \mathrm{C}$ CPMAS NMR (SiPL, ppm) $\delta: 4.0\left(\mathrm{POSiCH}_{3}\right)$.

${ }^{29} \mathrm{Si}$ CPMAS NMR (SiPL, ppm) $\delta: 25\left(\mathrm{POSiMe}_{3}\right),-94\left(\mathrm{ClSiO}_{3}\right)$, $-116\left(\mathrm{SiO}_{4}\right),-196\left(\mathrm{SiO}_{6}\right),-215\left(\mathrm{SiO}_{6}\right)$.

${ }^{31} \mathrm{P}$ MAS NMR (SiPL, ppm) $\delta:-49\left(\mathrm{P}(\mathrm{OSi} / \mathrm{Al}){ }_{4}\right)$.

${ }^{27} \mathrm{Al}$ MAS NMR (SiPL, ppm) $\delta: 89\left({ }^{[4]} \mathrm{Al}\right), 73\left({ }^{[4]} \mathrm{Al}\right), 55\left({ }^{[4]} \mathrm{Al}\right)$.

BET: $434 \mathrm{~m}^{2} \mathrm{~g}^{-1}$.

IR (SiC2SiPL, KBr, cm $\left.{ }^{-1}\right) \nu: 502 \mathrm{~m}, 701 \mathrm{vw}, 763 \mathrm{w}\left(\rho_{\mathrm{s}} \mathrm{SiCH}_{3}\right)$, $854 \mathrm{~m}\left(\rho_{\mathrm{as}} \mathrm{SiCH}_{3}\right), 1115 \mathrm{vs}(\nu \mathrm{Si}-\mathrm{O}-\mathrm{P}), 1258 \mathrm{~s}\left(\delta_{\mathrm{s}} \mathrm{SiCH}_{3}\right), 1410 \mathrm{vw}$ $\left(\delta_{\text {as }} \mathrm{CH}_{3}\right), 2918 \mathrm{vw}\left(\nu_{\mathrm{s}} \mathrm{CH}_{3}\right), 2965 \mathrm{~m}\left(\nu_{\text {as }} \mathrm{CH}_{3}\right)$.

${ }^{13} \mathrm{C}$ CPMAS NMR (SiC2SiPL, ppm) $\delta: 2.3\left(\mathrm{SiCH}_{2} \mathrm{CH}_{2} \mathrm{Si}+\right.$ $\mathrm{POSiCH}_{3}$ ).

${ }^{29} \mathrm{Si}$ CPMAS NMR (SiC2SiPL, ppm) $\delta:-71\left(\mathrm{CSiO}_{3}\right)$.

${ }^{31} \mathrm{P}$ MAS NMR (SiC2SiPL, ppm) $\delta:-52\left(\mathrm{P}(\mathrm{OSi} / \mathrm{Al})_{4}\right)$.

${ }^{27} \mathrm{Al}$ MAS NMR (SiC2SiPL, ppm) $\delta: 73\left({ }^{[4]} \mathrm{Al}\right), 57\left({ }^{[4]} \mathrm{Al}\right)$, $41\left({ }^{[5]} \mathrm{Al}\right)$.

BET: $458 \mathrm{~m}^{2} \mathrm{~g}^{-1}$.

Brønsted acidic catalysts (SiPB and SiC2SiPB). Xerogels were reacted with $\mathrm{POCl}_{3}$ in toluene with the elimination of $\mathrm{Me}_{3} \mathrm{SiCl}$ and $\mathrm{AcCl}(\mathrm{Cl}$ : residual organic group ratio $=3: 2)$ and then with water in THF with the elimination of $\mathrm{HCl}\left(\mathrm{H}_{2} \mathrm{O}\right.$ : residual $\mathrm{Cl}$ ratio $=1: 1$ ). The surface was covered with the $\mathrm{P}-\mathrm{OH}$ groups after this process. All reactions were carried out (solvents, times, temperatures) as described in Part 1.

IR (SiPB, KBr, cm $\left.{ }^{-1}\right) \nu: 497 \mathrm{~m}, 663 \mathrm{w}, 763 \mathrm{w}\left(\rho_{\mathrm{s}} \mathrm{SiCH}_{3}\right), 854 \mathrm{~m}$ $\left(\rho_{\text {as }} \mathrm{SiCH}_{3}\right), 1105$ vs $(\nu \mathrm{Si}-\mathrm{O}-\mathrm{P}), 1258 \mathrm{~s}\left(\delta_{\mathrm{s}} \mathrm{SiCH}_{3}\right), 2913 \mathrm{vw}$ $\left(\nu_{\mathrm{s}} \mathrm{CH}_{3}\right), 2965 \mathrm{~m}\left(\nu_{\text {as }} \mathrm{CH}_{3}\right)$.

${ }^{13} \mathrm{C}$ CPMAS NMR (SiPB, ppm) $\delta: 3.3\left(\mathrm{POSiCH}_{3}\right), 28.2$ $\left(\mathrm{CH}_{3} \mathrm{COO}\right.$ unident).

${ }^{29} \mathrm{Si}$ CPMAS NMR (SiPB, ppm) $\delta: 28\left(\mathrm{POSiMe}_{3}\right),-110\left(\mathrm{SiO}_{4}\right)$, $-213\left(\mathrm{SiO}_{6}\right)$.

${ }^{31} \mathrm{P}$ MAS NMR (SiPB, ppm) $\delta: \quad-41 \quad\left(\mathrm{P}(\mathrm{OSi})_{4}\right), \quad-30$ $\left(\mathrm{P}(\mathrm{OH})(\mathrm{OSi})_{3}\right),-19\left(\mathrm{P}(\mathrm{OH})_{2}(\mathrm{OSi})_{2}\right),-10\left(\mathrm{P}(\mathrm{OH})_{3}(\mathrm{OSi})\right)$.

BET: $117 \mathrm{~m}^{2} \mathrm{~g}^{-1}$.

IR (SiC2SiPB, KBr, cm $\left.{ }^{-1}\right) \nu: 497 \mathrm{~m}, 786 \mathrm{w}, 858 \mathrm{w}\left(\rho_{\text {as }} \mathrm{SiCH}_{3}\right)$, 1039 vs $(\nu \mathrm{Si}-\mathrm{O}-\mathrm{P}), 1262 \mathrm{~s}\left(\delta_{\mathrm{s}} \mathrm{SiCH}_{3}\right), 2913 \mathrm{vw}\left(\nu_{\mathrm{s}} \mathrm{CH}_{3}\right), 2960 \mathrm{vw}$ $\left(\nu\right.$ as $\left.\mathrm{CH}_{3}\right)$.

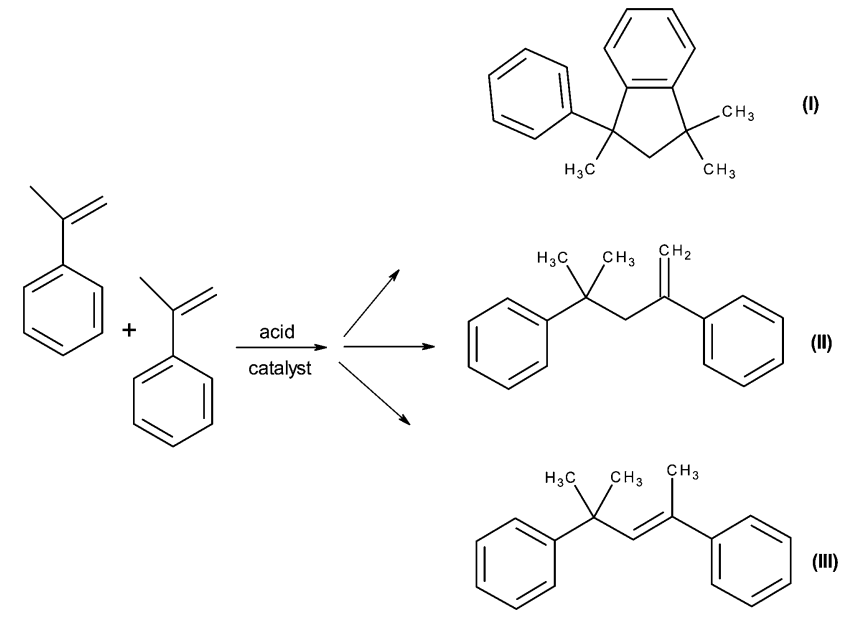

Scheme 1 Methylstyrene dimerization.

${ }^{13} \mathrm{C}$ CPMAS NMR (SiC2SiPB, ppm) $\delta: 0.8$ shoulder $\left(\mathrm{POSiCH}_{3}\right)$, $5.6\left(\mathrm{SiCH}_{2} \mathrm{CH}_{2} \mathrm{Si}\right) 24.5\left(\mathrm{CH}_{3} \mathrm{COO}\right.$ unident).

${ }^{29} \mathrm{Si}$ CPMAS NMR (SiC2SiPB, ppm) $\delta: 29\left(\mathrm{POSiMe}_{3}\right),-66$ $\left(\mathrm{CSiO}_{3}\right)$.

${ }^{31} \mathrm{P}$ MAS NMR (SiC2SiPB, ppm) $\delta:-35\left(\mathrm{O}=\mathrm{P}(\mathrm{OSi})_{3}\right),-23$ $\left(\mathrm{P}(\mathrm{OH})_{2}(\mathrm{OSi})_{2}\right)$.

BET: $172 \mathrm{~m}^{2} \mathrm{~g}^{-1}$.

\section{Catalytic test reactions}

Methylstyrene dimerization was recently utilized as a test reaction for the Brønsted acid catalysts under laboratory conditions in which superior results were achieved with phosphoric acid impregnated silica. ${ }^{23}$ Protocol: $4-5 \mathrm{wt} \%$ of catalyst was mixed with dry $\alpha$-methylstyrene and a stirred mixture was placed in an oil bath preheated to $90{ }^{\circ} \mathrm{C}$. After six hours the reaction mixture was allowed to cool down, the heterogeneous catalyst was filtered off and the mixture was diluted with toluene $(1: 20)$. GC-MS spectra of diluted samples were collected and the yields of methylstyrene dimers (I, II, III, Scheme 1) were estimated based on their intensities. The catalyst reusability was tested after separating it from the reaction mixture by centrifugation, washing three times with dry toluene $\left(10 \mathrm{~cm}^{3}\right)$, and drying in vacuo at room temperature. The dried catalyst was used again for the next $\alpha$-methylstyrene dimerization.

\section{Results and discussion}

\section{Chemical modification of silicophosphate xerogel surfaces}

Reactions with water. As already discussed, our parent materials (SiP, SiC2SiP) possess $10-20 \%$ of reactive $\equiv \mathrm{Si}-\mathrm{OAc}$ (AcO, acetoxy-) and $\equiv \mathrm{P}-\mathrm{OSiMe}_{3}$ (TMS, trimethylsilyl-) groups. There are two types of $\mathrm{SiO}_{6}$ units in the silicophosphate xerogels. One is formed by coordination of phosphoryl oxygens (-213 ppm) and the other by carboxylic oxygen of acetate $(-194 \mathrm{ppm}) .{ }^{18,19}$ It is clear from the comparison of IR spectra before and after hydrolysis that acetoxy groups are depleted (eqn (7)) in the course of the reaction as indicated by the diminished intensity of the 


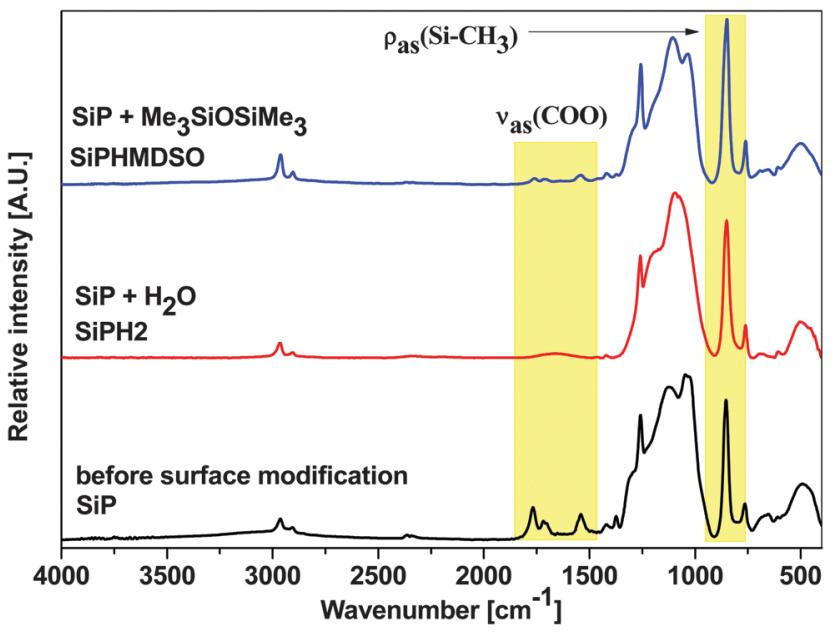

Fig. 1 Comparison of infrared spectra of parent xerogel (SiP), xerogel after hydrolysis (SiPH2), and after reaction with hexamethyldisiloxane (SiPHMDSO), KBr pellet.

absorption bands of COO stretches at 1768, 1721, and $1540 \mathrm{~cm}^{-1}$ (Fig. 1). ${ }^{18}$ The depletion of acetoxy groups was evidenced also in the ${ }^{13} \mathrm{C}$ and ${ }^{29} \mathrm{Si}$ CPMAS NMR spectra. Resonances of acetoxy groups are absent in the ${ }^{13} \mathrm{C}$ spectra and the $\mathrm{SiO}_{6}$ moieties with coordinated acetate oxygens disappear from the ${ }^{29} \mathrm{Si}$ spectra. ${ }^{18}$ It is also possible that the formed $\equiv \mathrm{Si}-\mathrm{OH}$ groups react further with $\equiv \mathrm{Si}-\mathrm{OAc}$ groups and condense to $\equiv \mathrm{Si}-\mathrm{O}-\mathrm{Si} \equiv$ bridges (eqn (8)) or eliminate water in homocondensation (eqn (9)).

$$
\begin{aligned}
& \equiv \mathrm{Si}-\mathrm{OAc}+\mathrm{H}_{2} \mathrm{O} \rightarrow \equiv \mathrm{Si}-\mathrm{OH}+\mathrm{AcOH} \\
& \equiv \mathrm{Si}-\mathrm{OH}+\mathrm{AcO}-\mathrm{Si} \equiv \rightarrow \equiv \mathrm{Si}-\mathrm{O}-\mathrm{Si} \equiv+\mathrm{AcOH} \\
& \equiv \mathrm{Si}-\mathrm{OH}+\mathrm{HO}-\mathrm{Si} \equiv \rightarrow \equiv \mathrm{Si}-\mathrm{O}-\mathrm{Si} \equiv+\mathrm{HOH}
\end{aligned}
$$

In contrast to the acetoxy groups, the $\equiv \mathrm{P}-\mathrm{OSiMe}_{3}$ groups are stable under these reaction conditions. The intensities of absorption bands characteristic for the $\mathrm{SiMe}_{3}$ moieties ${ }^{24}$ at 1258, 853, and $765 \mathrm{~cm}^{-1}$ do not change and also the signals of trimethylsilyloxy groups at -3.6 and $23.6 \mathrm{ppm}$ remain unchanged in the ${ }^{13} \mathrm{C}$ and ${ }^{29} \mathrm{Si}$ CPMAS NMR spectra, respectively.

If we consider the substitution of $\mathrm{CH}_{3} \mathrm{CO}$ groups with protons in terms of the mass of the samples, the weight should decrease. However the yields of the products are slightly higher than the starting masses of the xerogels (Table 1). In accordance with increasing mass of the samples the mass losses (TGA) increase after hydrolysis (Table 4). It is consistent with the suggestion that water could either be adsorbed onto the surface of the xerogels or hydrolyze the $\equiv \mathrm{Si}-\mathrm{O}-\mathrm{P} \equiv$ bonds as these are not hydrolytically stable ${ }^{25}$ (eqn (10)). We were able to distinguish between these two possibilities with the help of ${ }^{31} \mathrm{P}$ MAS NMR spectra. The parent xerogel displayed only one signal at $-45 \mathrm{ppm}\left(\mathrm{P}(\mathrm{OSi})_{4}\right)^{18}$ and this signal split after the reaction with water to several overlapping resonances with maxima at $-47,-36,-26$, and $-16 \mathrm{ppm}$ assigned to $\mathrm{P}(\mathrm{OSi})_{4-n}(\mathrm{OH})_{n}$ phosphorus environments, where $n=0-3 .^{26,27}$ The least shielded resonance at $-8 \mathrm{ppm}$ of a low intensity was assigned to $\mathrm{O}=\mathrm{P}(\mathrm{OH})_{2}(\mathrm{OSi})$ moieties. ${ }^{26,27}$ Based on these NMR data we conclude that hydrolysis of the skeletal $\equiv \mathrm{Si}-\mathrm{O}-\mathrm{P} \equiv$ bonds takes place (eqn (10)) to a large extent and the structure of silicophosphate xerogels collapses. The more water used for the reaction, the more intensive hydrolysis occurred $-{ }^{31} \mathrm{P}$ MAS NMR spectra showed more downfield signals with higher intensities coming from the higher number of $\mathrm{HO}-\mathrm{P} \equiv$ groups (Fig. S1, ESI $\dagger$ ). It is also noteworthy that while the skeletal $\equiv \mathrm{Si}-\mathrm{O}-\mathrm{P} \equiv$ groups undergo facile hydrolysis, the surface $\mathrm{Me}_{3} \mathrm{Si}-\mathrm{O}-\mathrm{P} \equiv$ bonds are stable (Fig. 1 ).

$\equiv \mathrm{Si}-\mathrm{O}-\mathrm{P} \equiv+\mathrm{H}_{2} \mathrm{O} \rightarrow \equiv \mathrm{Si}-\mathrm{OH}+\mathrm{HO}-\mathrm{P} \equiv$

The reaction in eqn (10) should yield strongly acidic $\equiv \mathrm{P}-\mathrm{OH}$ groups. This was confirmed by IR spectroscopy of pyridinetreated samples. Non-hydrolyzed SiP samples did not show any uptake of pyridine. In contrast, absorption bands at 1488, 1537, and $1640 \mathrm{~cm}^{-1}$ were observed for all samples reacted with water (Fig. S2, ESI $\dagger$ ). These wavenumbers are typical for pyridinium cations suggesting that Brønsted acid sites were formed during the hydrolysis in accordance with our previous observations.

The breakdown of the silicophosphate (SiP) structure by hydrolysis of skeletal $\equiv \mathrm{Si}-\mathrm{O}-\mathrm{P} \equiv$ linkages (eqn (10)) results in significant losses of SA (Table 4). Thus we obtain acidic but nonporous materials. Hybrid silicophosphates (SiC2SiP) displayed better results in terms of porosity after the reaction with water. They lose acetoxy groups and a large amount of $\equiv \mathrm{Si}-\mathrm{O}-$ $\mathrm{P} \equiv$ bonds similar to parent SiP xerogels; however, they keep some surface area even if an equimolar amount of water $(100 \%$ relative to residual organic groups) is used in the hydrolysis (SiC2SiPH1, Table 4). Considering the possible application of our materials, we have to be aware of their facile hydrolysis. Therefore they should be used under anhydrous or better still aprotic conditions. As we have shown recently, silicophosphates show only limited resistance to methanol since this reaction produces acetic acid in the first step and methylacetate and water in the second step. The esterification reaction is strongly shifted to the side of methylacetate, because water is promptly consumed in the hydrolysis of the $\mathrm{Si}-\mathrm{O}-\mathrm{P}$ backbone. $^{18}$

Reactions with hexamethyldisiloxane. In the case of reactions with hexamethyldisiloxane, the acetoxy groups displayed significant reactivity and were almost quantitatively substituted by trimethylsilyloxy groups (eqn (11)) if we used 50\% of HMDSO relative to residual organic groups (both acetoxy and trimethylsilyloxy). The volatile product of the reaction - trimethylsilylacetate - was identified by GC-MS and the surface bound $\equiv \mathrm{Si}-\mathrm{OSiMe}_{3}$ groups were evidenced by a new resonance at 13.6 ppm in the ${ }^{29} \mathrm{Si}$ CPMAS NMR spectrum. At the same time the intensity of vibrations of acetoxy groups diminished in the IR spectra (Fig. 1), and their resonances were absent in the ${ }^{13} \mathrm{C}$ CPMAS NMR spectra. Finally the signal of $\mathrm{SiO}_{6}$ moieties at -196 ppm coming from the additional coordination of carboxyl groups disappeared in the ${ }^{29} \mathrm{Si}$ CPMAS NMR spectrum.

The substitution of $\mathrm{SiO}_{6}$-coordinated bridging acetoxy groups with terminal trimethylsilyloxy groups caused a significant decrease of surface area to half of its value. A similar effect 
has been observed after the methanolysis of silicophosphate xerogel, where the $\mathrm{CH}_{3} \mathrm{COO}$ groups were exchanged with methoxy groups. ${ }^{18}$ We also tried methyl ${ }^{t}$ butylether for the elimination of acetoxy-groups. In all cases the resonance of $\mathrm{SiO}_{6}$ at $-196 \mathrm{ppm}$ diminished, $\mathrm{CH}_{3} \mathrm{COO}$ groups were effectively substituted and samples lost from 45 to $65 \%$ of SA and pore volume in comparison to original xerogels. Therefore we conclude that the bridging nature of acetates is very important for the porous structure of these silicophosphate xerogels (SiP samples).

The silicophosphate skeleton is also not resistant towards HMDSO attack and new $\equiv \mathrm{Si}-\mathrm{OSiMe}_{3}$ and $\equiv \mathrm{P}-\mathrm{OSiMe}_{3}$ groups are formed (eqn (12)) if excess of HMDSO is used for the reaction. SiP heated in neat HMDSO completely decomposed and provided a mixture of $\mathrm{OP}\left(\mathrm{OSiMe}_{3}\right)_{3}$ and $\mathrm{Si}\left(\mathrm{OSiMe}_{3}\right)_{4}$. The hybrid SiC2SiP xerogel showed similar reactivity.

$\equiv \mathrm{Si}-\mathrm{OAc}+\mathrm{Me}_{3} \mathrm{SiOSiMe}_{3} \rightarrow \equiv \mathrm{Si}-\mathrm{OSiMe}_{3}+\mathrm{AcOSiMe}_{3}$

$\equiv \mathrm{Si}-\mathrm{O}-\mathrm{P} \equiv+\mathrm{Me}_{3} \mathrm{SiOSiMe}_{3} \rightarrow \equiv \mathrm{Si}-\mathrm{OSiMe}_{3}+\mathrm{Me}_{3} \mathrm{SiO}-\mathrm{P} \equiv$

Reactions with $\mathrm{SiCl}_{4}$ and $\mathrm{POCl}_{3}$. Silicon tetrachloride reacted with both acetoxy and trimethylsilyloxy groups (the ratio of $\mathrm{Cl}$ to the sum of $\mathrm{AcO}$ and $\mathrm{Me}_{3} \mathrm{Si}$ was $1: 1$ ), but with TMS to a larger extent. The organic products were trimethylchlorosilane and acetylchloride according to GC-MS (eqn (13) and (14)). The intensities of the signals of $\mathrm{AcO}$ and $\mathrm{Me}_{3} \mathrm{Si}$ groups after the reaction were significantly reduced in $\mathrm{IR},{ }^{13} \mathrm{C}$, and ${ }^{29} \mathrm{Si}$ CPMAS $\mathrm{NMR}$ spectra. At the same time, new signals of $\mathrm{Cl}_{2} \mathrm{SiO}_{2}$ and $\mathrm{ClSiO}_{3}$ moieties were observed in the ${ }^{29} \mathrm{Si}$ CPMAS NMR spectra (Fig. 2), terminal $\mathrm{Cl}_{3} \mathrm{SiO}$ groups were not observed. However, these were observed at $-43 \mathrm{ppm}$ when the reaction was performed at a 1.4:1 ratio of $\mathrm{Cl}$ : residual organic groups. Silicon tetrachloride thus acted as a significant cross-linking agent and this was reflected in a substantial and reproducible increase of SA from 487 to $865 \mathrm{~m}^{2} \mathrm{~g}^{-1}$. Reactions that are consistent with these observations are summarized in eqn (15)-(18).

$$
\begin{aligned}
& \equiv \mathrm{Si}-\mathrm{OAc}+\mathrm{SiCl}_{4} \rightarrow \equiv \mathrm{Si}-\mathrm{O}-\mathrm{SiCl}_{3}+\mathrm{AcOCl} \\
& \equiv \mathrm{P}-\mathrm{O}-\mathrm{SiMe}_{3}+\mathrm{SiCl}_{4} \rightarrow \equiv \mathrm{P}-\mathrm{O}-\mathrm{SiCl}_{3}+\mathrm{Me}_{3} \mathrm{SiCl} \\
& \equiv \mathrm{P}-\mathrm{O}-\mathrm{SiCl}_{3}+\mathrm{Me}_{3} \mathrm{Si}-\mathrm{O}-\mathrm{P} \equiv \rightarrow \equiv \mathrm{P}-\mathrm{O}-\mathrm{Si}\left(\mathrm{Cl}_{2}\right)-\mathrm{O}-\mathrm{P} \equiv+\mathrm{Me}_{3} \mathrm{SiCl}
\end{aligned}
$$

$\equiv \mathrm{Si}-\mathrm{O}-\mathrm{SiCl}_{3}+\mathrm{AcO}-\mathrm{Si} \equiv \rightarrow \equiv \mathrm{Si}-\mathrm{O}-\mathrm{Si}\left(\mathrm{Cl}_{2}\right)-\mathrm{O}-\mathrm{Si} \equiv+\mathrm{AcOCl}$

$\equiv \mathrm{P}-\mathrm{O}-\mathrm{SiCl}_{3}+\mathrm{AcO}-\mathrm{Si} \equiv \rightarrow \equiv \mathrm{P}-\mathrm{O}-\mathrm{Si}\left(\mathrm{Cl}_{2}\right)-\mathrm{O}-\mathrm{Si} \equiv+\mathrm{AcOCl}$

$\equiv \mathrm{Si}-\mathrm{O}-\mathrm{SiCl}_{3}+\mathrm{Me}_{3} \mathrm{Si}-\mathrm{O}-\mathrm{P} \equiv \rightarrow \equiv \mathrm{Si}-\mathrm{O}-\mathrm{Si}\left(\mathrm{Cl}_{2}\right)-\mathrm{O}-\mathrm{P} \equiv+\mathrm{Me}_{3} \mathrm{SiCl}$

The facile condensation between $\equiv \mathrm{P}-\mathrm{OSiMe}_{3}$ and $\mathrm{Si}-\mathrm{Cl}$ groups (eqn (14)) could be potentially used for anchoring of various organic functionalities through alkyl- or arylchlorosilanes. As an example, we reacted benzyltrichlorosilane, $\mathrm{PhCH}_{2} \mathrm{SiCl}_{3}$, with silicophosphate xerogels providing samples

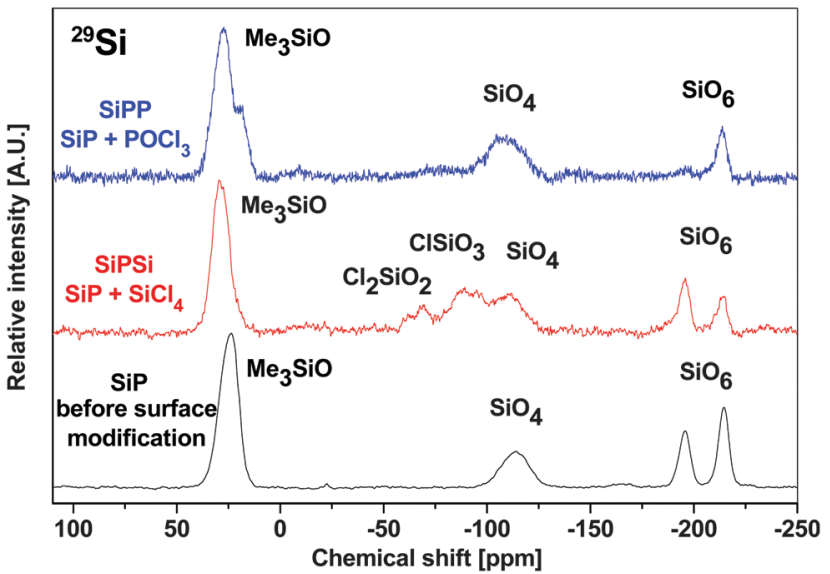

Fig. $2{ }^{29} \mathrm{Si}$ CPMAS NMR spectra of xerogels before surface modification (parent $\mathrm{SiP}$ ) and after reaction with silicon tetrachloride (SiPSi) and phosphoryl trichloride (SiPP).

(SiPPhCH2Si) with similar surface areas and $\mathrm{O}_{3} \mathrm{SiCH}_{2} \mathrm{Ph}$ groups bound to the surface as evidenced by ${ }^{13} \mathrm{C}$ and ${ }^{29} \mathrm{Si}$ CP MAS NMR spectra (Fig. S3, ESI $\dagger$ ). The $\mathrm{Si}-\mathrm{C}$ bonds are stable under the reaction conditions. This reaction manifold provides us various possibilities to modify the surface of porous inorganic support.

While phosphoryl trichloride reacted with both $\mathrm{AcO}$ and TMS groups similar to $\mathrm{SiCl}_{4}$ (eqn (19) and (20)), the overall reactivity of $\mathrm{POCl}_{3}$ towards SiP samples was lower. When equimolar amounts of chlorine atoms of $\mathrm{POCl}_{3}$ with respect to residual groups were used, unreacted phosphoryl trichloride was observed among volatiles by GC-MS together with acetylchloride and trimethylchlorosilane. The acetoxy groups were in this case more effectively depleted in comparison to the reaction with silicon tetrachloride (Fig. 2). We did not observe any indication of ligand scrambling between $\equiv \mathrm{Si}-\mathrm{OAc}$ and $\mathrm{POCl}_{3}$ in the ${ }^{29} \mathrm{Si}$ CPMAS NMR spectra (Fig. 2). The surface area remained virtually unchanged $\left(483 \mathrm{~m}^{2} \mathrm{~g}^{-1}\right)$.

As a proof of successful anchoring of $\mathrm{POCl}_{3}$ onto the surface of silicophosphate xerogels, a new signal at -31 ppm was observed in ${ }^{31} \mathrm{P}$ MAS NMR spectra which could be assigned to $\mathrm{O}=\mathrm{P}(\mathrm{OSi} / \mathrm{P})_{3}$ or $\mathrm{ClP}(\mathrm{OSi} / \mathrm{P})_{3}$ moieties (Fig. 3). The first two steps of condensation reactions leading to moieties with this shift are described in eqn (19)-(24). The condensation can proceed even one step further to provide $\mathrm{O}=\mathrm{P}(\mathrm{OSi} / \mathrm{P})_{3}$ groups with a chemical shift of $c a$. $-30 \mathrm{ppm}$ in ${ }^{31} \mathrm{P}$ MAS NMR. ${ }^{19}$ The second possible reaction providing phosphorus atoms with a similar chemical shift is the coordination of free phosphoryl oxygen to the $\mathrm{Si}$ atoms. This possible reaction would increase the coordination number of silicons and produce $\mathrm{ClP}(\mathrm{OSi} / \mathrm{P})_{3}$ moieties. The signals in the ${ }^{31} \mathrm{P}$ MAS NMR spectrum of the SiPP sample are however broad and overlapping which prevents a more precise description of the new surface groups (Fig. 3).

$\equiv \mathrm{Si}-\mathrm{OAc}+\mathrm{P}(\mathrm{O}) \mathrm{Cl}_{3} \rightarrow \equiv \mathrm{Si}-\mathrm{O}-\mathrm{P}(\mathrm{O}) \mathrm{Cl}_{2}+\mathrm{AcOCl}$

$\equiv \mathrm{P}-\mathrm{O}-\mathrm{SiMe}_{3}+\mathrm{P}(\mathrm{O}) \mathrm{Cl}_{3} \rightarrow \equiv \mathrm{P}-\mathrm{O}-\mathrm{P}(\mathrm{O}) \mathrm{Cl}_{2}+\mathrm{Me}_{3} \mathrm{SiCl}$ 


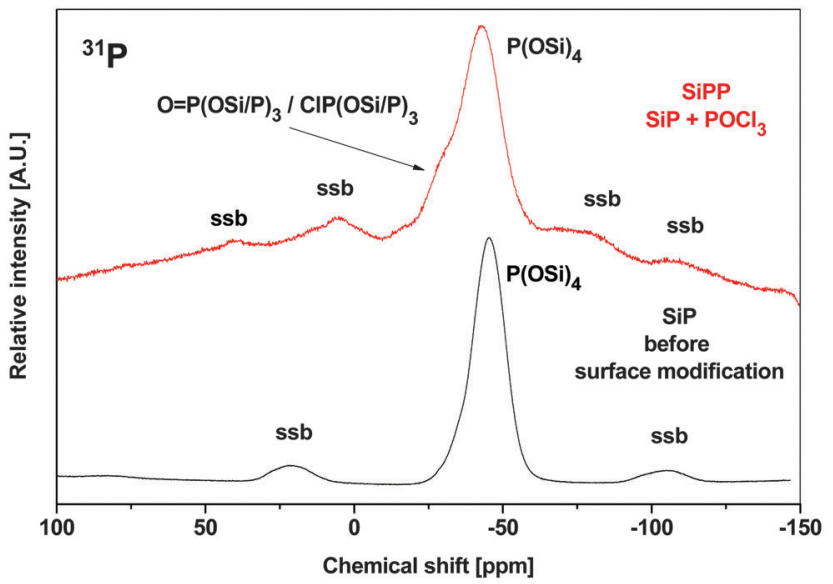

Fig. $3{ }^{31} \mathrm{P}$ MAS NMR spectra of xerogels before surface modification (parent SiP) and after reaction with phosphoryl trichloride (SiPP).

$$
\begin{aligned}
\equiv & \mathrm{P}-\mathrm{O}-\mathrm{P}(\mathrm{O}) \mathrm{Cl}_{2}+\mathrm{Me}_{3} \mathrm{Si}-\mathrm{O}-\mathrm{P} \equiv \\
& \rightarrow \equiv \mathrm{P}-\mathrm{O}-\mathrm{P}(\mathrm{O})(\mathrm{Cl})-\mathrm{O}-\mathrm{P} \equiv+\mathrm{Me}_{3} \mathrm{SiCl} \\
\equiv & \mathrm{Si}-\mathrm{O}-\mathrm{P}(\mathrm{O}) \mathrm{Cl}_{2}+\mathrm{AcO}-\mathrm{Si} \equiv \\
& \rightarrow \equiv \mathrm{Si}-\mathrm{O}-\mathrm{P}(\mathrm{O})(\mathrm{Cl})-\mathrm{O}-\mathrm{Si} \equiv+\mathrm{AcOCl} \\
\equiv \mathrm{P}-\mathrm{O}-\mathrm{P}(\mathrm{O}) \mathrm{Cl}_{2}+\mathrm{AcO}-\mathrm{Si} \equiv & \rightarrow \equiv \mathrm{P}-\mathrm{O}-\mathrm{P}(\mathrm{O})(\mathrm{Cl})-\mathrm{O}-\mathrm{Si} \equiv+\mathrm{AcOCl} \\
& \rightarrow \mathrm{Si}-\mathrm{O}-\mathrm{P}(\mathrm{O}) \mathrm{Cl}_{2}+\mathrm{Me}{ }_{3} \mathrm{Si}-\mathrm{O}-\mathrm{P} \equiv \\
& \rightarrow \equiv \mathrm{Si}-\mathrm{O}-\mathrm{P}(\mathrm{O})(\mathrm{Cl})-\mathrm{O}-\mathrm{P} \equiv+\mathrm{Me}_{3} \mathrm{SiCl}
\end{aligned}
$$

The efficiency of anchoring Si and P compounds on the xerogel surfaces was proven by elemental analyses of phosphorus, total acidity, and argentometric analyses of $\mathrm{Cl}$ (Table 2). All experimental data are in reasonable agreement with theoretical values confirming the grafting of xerogel surfaces with $\mathrm{SiCl}_{4}$ and $\mathrm{POCl}_{3}$.

Reactions with $\mathrm{AlMe}_{3}, \mathbf{A l}\left(\mathrm{NMe}_{2}\right)_{3}$, and $\mathbf{A l C l}_{3}$. The chemical modification of these silicophosphate gels with several aluminum compounds was explored as a potential source of Lewis acidic tetrahedral Al sites on the surface of silicophosphate supports. Volatile products of all three reactions contained expected organic compounds (acetone and tetramethylsilane in the case of $\mathrm{AlMe}_{3}$,
Table 4 Relative amounts of water used in the reactions, mass losses during TGA, and surface areas of the products

\begin{tabular}{lccc}
\hline Sample & $n_{\mathrm{H}_{2} \mathrm{O}}{ }^{a}[\%]$ & $\mathrm{ML}_{\exp }{ }^{b}[\%]$ & $\mathrm{SA}\left[\mathrm{m}^{2} \mathrm{~g}^{-1}\right]$ \\
\hline SiPH1 & 10 & 37.7 & 279 \\
SiPH2 & 50 & 40.6 & Nonporous $^{c}$ \\
SiPH3 & 100 & 46.4 & Nonporous \\
SiC2SiPH1 & 100 & 28.1 & 42 \\
\multicolumn{2}{l}{ Relative to residual organic groups. } & ${ }^{b}$ Mass loss at $1000{ }^{\circ} \mathrm{C}$ in air with \\
the heating ramp of $5 \mathrm{~K} \mathrm{~min}^{-1} \cdot{ }^{c}$ SA below $10 \mathrm{~m}^{2} \mathrm{~g}^{-1}$.
\end{tabular}

dimethylacetamide and dimethyl(trimethylsilyl)amine in the case of $\mathrm{Al}\left(\mathrm{NMe}_{2}\right)_{3}$, and trimethylchlorosilane for $\left.\mathrm{AlCl}_{3}\right)$. These compounds are obviously the products of condensation of residual

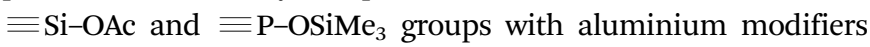
and attest to the formation of new $\mathrm{Al}-\mathrm{O}-\mathrm{Si}$ and $\mathrm{Al}-\mathrm{O}-\mathrm{P}$ bonds on the xerogel surfaces. The anchoring of $\mathrm{Al}$ atoms onto the surface of xerogels was confirmed by elemental analysis of the samples, where $\mathrm{Si}, \mathrm{P}$, and $\mathrm{Al}$ values were in good agreement with theoretical values (Table 3). Although the reactions afforded expected organic byproducts and $\mathrm{Al}$ atoms were grafted onto the surface, the inorganic products displayed significant differences in the structure and coordination number of $\mathrm{Al}$ atoms. This was caused by the different propensity of Al precursors towards ligand scrambling (see below).

Al-Me moieties are present in samples treated with $\mathrm{AlMe}_{3}$ as suggested by the IR and ${ }^{13} \mathrm{C}$ CPMAS NMR spectra of the SiPAl1 sample, where we observed absorption bands at 689, 1193, and $2937 \mathrm{~cm}^{-1}$ assigned to $\nu_{\text {as }} \mathrm{AlC}_{3}, \delta_{\mathrm{s}} \mathrm{AlCH}_{3}$, and $\nu_{\text {as }} \mathrm{AlCH}_{3}$ vibrations ${ }^{28}$ and a ${ }^{13} \mathrm{C}$ resonance of shielded methyl groups bound to $\mathrm{Al}$ at $-7.9 \mathrm{ppm}$. However a significant amount of AlMe groups obviously exchanged with residual acetoxy groups (eqn (25)). This reaction is consistent with ${ }^{29} \mathrm{Si}$ CPMAS NMR data, where a composite broad signal of $\mathrm{Si}(\mathrm{OP})_{4-n}(\mathrm{OAc})_{n}$ moieties $(n=0-3)$ in the SiP sample observed at $-111 \mathrm{ppm}$ split into signals for $\mathrm{OSiMe}_{3}, \mathrm{O}_{2} \mathrm{SiMe}_{2}, \mathrm{O}_{3} \mathrm{SiMe}$, and $\mathrm{SiO}_{4}$ groups at $17,-4,-64$, and $-115 \mathrm{ppm}$, respectively, in the spectrum of SiPAl1 (Fig. S4, ESI $\dagger$ ). Simultaneously the $\mathrm{SiO}_{6}$ signal arising from the additional coordination of carboxyl oxygens of the AcO groups at $-196 \mathrm{ppm}$ disappeared. Finally the substitution of methyl groups at $\mathrm{Al}$ with bidentate acetoxy groups (eqn (25)) leads to a high coordination number for $\mathrm{Al}$ as evidenced by a signal at $-7 \mathrm{ppm}$ in the ${ }^{27} \mathrm{Al}$ MAS NMR spectra assigned to $\mathrm{Al}$

Table 2 Experimentally obtained and calculated $\mathrm{P}$ content from ICP, NaOH consumptions $\left(\mathrm{HCl}+\mathrm{HOAC}+\mathrm{H}_{3} \mathrm{PO}_{4}\right)$ and $\mathrm{Cl}$ content from argentometry of SiPSi and SiPP samples

\begin{tabular}{lllllll}
\hline Sample & $w(\mathrm{P})_{\exp }(\%)$ & $w(\mathrm{P})_{\text {calc }}(\%)$ & $n_{\text {exp }} \mathrm{NaOH}\left(\mathrm{mmol} \mathrm{g}^{-1}\right)$ & $n_{\text {calc }} \mathrm{NaOH}\left(\mathrm{mmol} \mathrm{g}^{-1}\right)$ & $n_{\exp } \mathrm{Cl}\left(\mathrm{mmol} \mathrm{g}^{-1}\right)$ & $n_{\text {calc }} \mathrm{Cl}\left(\mathrm{mmol} \mathrm{g}^{-1}\right)$ \\
\hline SiPSi & 15.7 & 17.7 & 15.0 & 16.3 & 2.90 & 2.87 \\
SiPP & 19.5 & nd & 14.1 & 15.4 & 1.12 & nd
\end{tabular}

Table 3 ICP-OES elemental analysis of P, Al, and Si contents. Comparison with theoretical values

\begin{tabular}{lllllll}
\hline Sample & $w(\mathrm{P})_{\exp }(\%)$ & $w(\mathrm{P})_{\text {calc }}(\%)$ & $w(\mathrm{Al})_{\exp }(\%)$ & $w(\mathrm{Al})_{\text {calc }}(\%)$ & $w(\mathrm{Si})_{\exp }(\%)$ & $w(\mathrm{Si})_{\text {calc }}(\%)$ \\
\hline SiPAl1 & 13.4 & 14.9 & 5.42 & 6.24 & 9.80 & 10.1 \\
SiPAl2 & 12.0 & 11.7 & 4.72 & 4.83 & 9.39 & 7.97 \\
SiPAl3 & 12.0 & 11.6 & 6.50 & 5.60 & 9.39
\end{tabular}




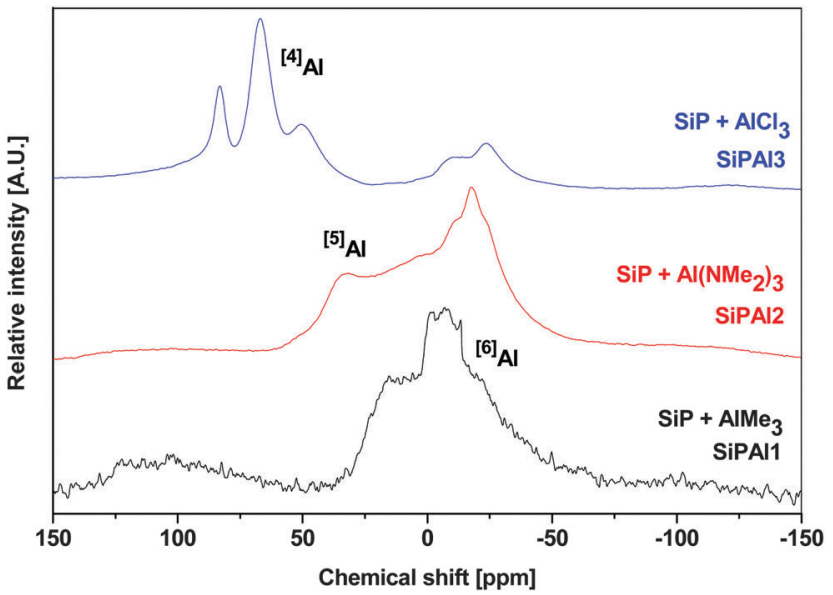

Fig. 4 Comparison of the ${ }^{27} \mathrm{Al}$ MAS NMR spectra of xerogels after reaction with aluminium compounds.

atoms in 6-fold coordination (Fig. 4). ${ }^{29}$ As a consequence of the total collapse of the structure the product was nonporous.

$\equiv \mathrm{Si}-\mathrm{OAc}+\mathrm{AlMe}_{3} \rightarrow \equiv \mathrm{Si}-\mathrm{Me}+\mathrm{AlMe}_{2} \mathrm{OAc}$

Better results were achieved in reactions with $\mathrm{Al}\left(\mathrm{NMe}_{2}\right)_{3}$. The ${ }^{29} \mathrm{Si}$ CPMAS NMR spectra of the SiPAl2 sample displayed features of both ligand exchange $\left(-68.5 \mathrm{ppm},\left(\mathrm{Me}_{2} \mathrm{~N}\right)_{2} \mathrm{SiO}_{2}\right.$, $\left.0.4 \mathrm{ppm}, \mathrm{AlOSiMe}_{3}\right)^{30-32}$ and condensation $\left(-90.0 \mathrm{ppm}, \mathrm{SiO}_{4}\right)$. The downfield shift of the $\mathrm{SiO}_{4}$ resonance with respect to silica $\left(-110.0 \mathrm{ppm}, \mathrm{SiO}_{4}\right)$ points to the formation of $\mathrm{Si}-\mathrm{O}-\mathrm{Al}$ bonds. $^{33,34}$ The signal at $-90.0 \mathrm{ppm}$ could be increased in intensity by ligand exchange $\left(\mathrm{Me}_{2} \mathrm{NSiO}_{3}\right)$ as well. ${ }^{30,32}$ The extent of ligand exchange was significantly smaller than in the case of the SiPAl1 sample and was comparable to the extent of condensation according to the ${ }^{29} \mathrm{Si}$ CPMAS NMR spectra. This was reflected in the ${ }^{27} \mathrm{Al}$ MAS NMR spectra, where hexacoordinated $\mathrm{Al}$ atoms were accompanied by pentacoordinated $\mathrm{Al}$ (Fig. 4). ${ }^{29}$ The surface area decreased slightly to $375 \mathrm{~m}^{2} \mathrm{~g}^{-1}$.

Aluminium trichloride provided the best results. We observed little evidence of ligand exchange by a signal of low intensity at $-91 \mathrm{ppm}$ in ${ }^{29} \mathrm{Si}$ CPMAS NMR spectra. This signal is probably not due to $\mathrm{SiO}_{4}$ centers associated with aluminium because no acetylchloride is eliminated during the reaction and therefore no Si-O-Al bridges are formed. Five signals were observed in the ${ }^{27} \mathrm{Al}$ MAS NMR spectrum of the SiPAl3 sample: two resonances of hexacoordinated $\mathrm{Al}$ atoms at $-23 \mathrm{ppm}$ and $-7 \mathrm{ppm}$ and three peaks of tetracoordinated $\mathrm{Al}$ atoms at 51,67 , and 83 ppm (Fig. 4). These three signals are typical for all our samples grafted with $\mathrm{AlCl}_{3}$ and we suggest that they come from $\mathrm{AlO}_{4}, \mathrm{AlO}_{3} \mathrm{Cl}$ and $\mathrm{AlO}_{2} \mathrm{Cl}_{2}$ moieties. ${ }^{29}$ The surface area increased from 403 to $469 \mathrm{~m}^{2} \mathrm{~g}^{-1}$. For these reasons $\mathrm{AlCl}_{3}$ was identified as a promising functionalizing reagent for synthesizing Lewis acidic and porous materials using silicophosphates as supports.

The residual acetoxy groups obviously play an important role in the increasing coordination number of $\mathrm{Al}$ atoms. The region between 1400 and $1800 \mathrm{~cm}^{-1}$, where absorption bands of COO valence vibrations are located, was always significantly different after the grafting of Al compounds. This was accompanied also by the changes in the ${ }^{13} \mathrm{C}$ CPMAS NMR spectra. For example, a new absorption band of the carboxyl group was observed at $1602 \mathrm{~cm}^{-1}$ in the IR spectra of the SiPAl3 sample and at the same time a new signal of carboxyl groups was identified at $181.6 \mathrm{ppm}$ in the ${ }^{13} \mathrm{C}$ CPMAS NMR spectra (in comparison with parent SiP xerogel). The wavenumber of the absorption band as well as the shift in the NMR spectrum suggest that the observed carboxyl group is bidentate. ${ }^{35,36}$ Acetoxy groups evidently coordinated to aluminium atoms causing its hypercoordination $\left({ }^{27} \mathrm{Al}\right.$ MAS NMR, Fig. 4) and shifting the parent signals in both IR and NMR spectra. Therefore we conclude that the acetoxy groups have a negative influence on the Lewis acidity of the products.

Preparation of solid acid catalysts. As already mentioned, the reaction of silicophosphate xerogels with water provided nonporous powders possessing Brønsted acidic $\equiv \mathrm{P}-\mathrm{OH}$ groups. These, however, may not be accessible because of the low surface area of samples. The only sample which retained porosity was the SiC2SiPH1 hybrid. Preliminary tests of silicophosphate samples in methylstyrene dimerization showed that both Brønsted acidity and porosity are very important for catalytic activity - the hybrid sample SiC2SiPH1 displayed a conversion of $33 \%$ whereas the nonporous sample SiPH2 converted less than $1 \%$ of methylstyrene (Table 5). The SiP sample without modification with water did not show any catalytic activity as expected. Therefore we focused on the synthesis of porous samples with high numbers of $\equiv \mathrm{P}-\mathrm{OH}$ groups on the surface. This was achieved by grafting of xerogels with phosphoryl trichloride and subsequent hydrolysis of the $\mathrm{P}-\mathrm{Cl}$ bonds (samples SiPB and SiC2SiPB, Table 5).

The samples SiPB and SiC2SiPB synthesized by a two-step protocol (see Experimental) showed both high acidity and surface areas (Table 5). It is noteworthy that even if the average number of $\mathrm{OH}$ groups on phosphorus is lower for these two samples, their overall acidity is higher as indicated by high pyridine uptakes. The acidity is unambiguously of Brønsted type as confirmed by the presence of the absorption bands of the pyridinium cation in the IR spectrum of the SiPB sample after pyridine adsorption (Fig. 5). This is caused by a large amount of phosphorus atoms on the surface of these samples after their grafting with $\mathrm{POCl}_{3}$.

These two samples displayed a high catalytic activity in $\alpha$-methylstyrene dimerization (Table 5) similar to silica impregnated

Table 5 Acidity, porosity, and catalytic properties of silicophosphate xerogels

\begin{tabular}{|c|c|c|c|c|}
\hline Sample & $\mathrm{SiPH} 2$ & SiC2SiPH1 & SiPB & SiC2SiPB \\
\hline Pyridine uptake $\left(\mathrm{mmol} \mathrm{g}^{-1}\right)$ & 1.16 & 2.58 & 1.70 & 2.77 \\
\hline $\begin{array}{l}\text { Average number of } \mathrm{OH} \text { groups } \\
\text { on } \mathrm{P}\end{array}$ & 1.6 & 1.6 & 1.1 & 0.9 \\
\hline $\mathrm{SA}\left(\mathrm{m}^{2} \mathrm{~g}^{-1}\right)$ & Nonporous & 42 & 117 & 172 \\
\hline Microporosity $^{a}(\%)$ & - & 0 & 56 & 0 \\
\hline Conversion (\%) & $<1$ & 33 & 99 & 99 \\
\hline Selectivity (\%) Isomer I & - & 1 & 14 & $<1$ \\
\hline Selectivity (\%) Isomer II & - & 82 & 29 & 92 \\
\hline Selectivity (\%) Isomer III & - & 17 & 57 & 8 \\
\hline
\end{tabular}

${ }^{a} V_{\text {micro }} / V_{\text {total }}, V_{\text {micro }}$ determined by $t$-plot analysis, $V_{\text {total }}$ estimated at $p / p_{0}=0.98$. 


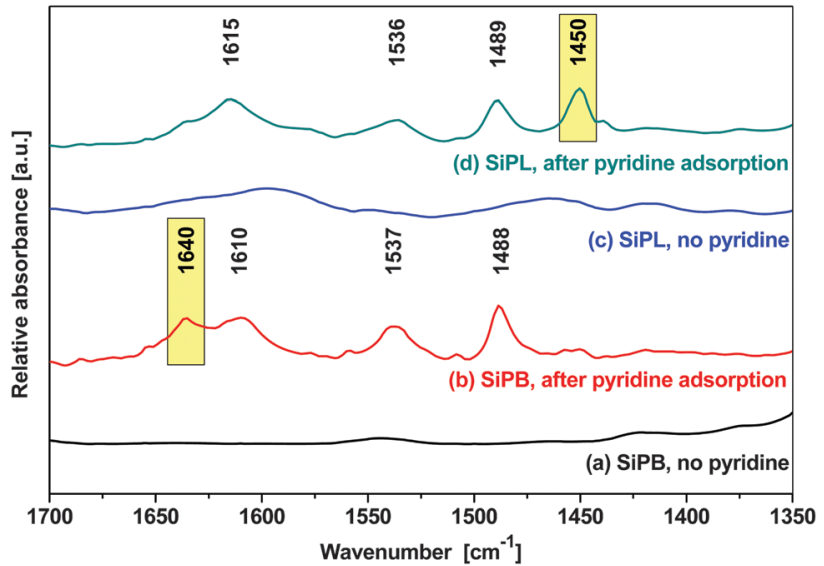

Fig. 5 IR spectra of the SiPB sample (a), SiPB after pyridine adsorption (b), SiPL (c), and SiPL after pyridine adsorption (d).

with phosphoric acid. ${ }^{23}$ It is notable that selectivity differs significantly according to the pore sizes and structure of silicophosphate samples - microporous xerogels based on the parent SiP silicophosphates reached moderate selectivity for 2-pentene (dimer III); however, mesoporous hybrid xerogels (SiC2SiP) showed a high selectivity for 1-pentene (dimer II) (Table 5). Thus, together with our previous catalytic results achieved on mesoporous nanocrystalline $\mathrm{Si}_{5} \mathrm{P}_{6} \mathrm{O}_{25}$, we developed heterogeneous catalysts for $\alpha$-methylstyrene dimerization with excellent conversions and moderate to excellent selectivities toward all possible methylstyrene dimers (I-III). ${ }^{37}$

Both catalysts can be reused without regeneration and maintain very high methylstyrene conversions in the second and third catalytic run (98 and 96\% for SiPB, 97 and $94 \%$ for SiC2SiPB). The selectivity after two catalytic cycles decreased by $8 \%$ for 2 -pentene by the SiPB sample and by $12 \%$ for 1 -pentene by the SiC2SiPB catalyst. The heterogeneous nature of catalytic action is supported by the absence of phosphorus in the reaction solution (ICP-OES). The described two-step synthesis uses the knowledge of surface reactions on our silicophosphate xerogels to transform them into efficient and selective Brønsted acidic catalysts.

Finally we have also attempted to prepare porous Lewis acidic xerogels. As mentioned above, we found that acetoxy groups coordinate to $\mathrm{Al}$ atoms thus decreasing their Lewis acidity. However acetoxy groups can be depleted in the reaction with hexamethyldisiloxane. Therefore the first step of synthesis was the removal of acetoxy groups by hexamethyldisiloxane and subsequent grafting with $\mathrm{AlCl}_{3}$, which displayed the best results among the tested aluminum precursors. This synthetic protocol was performed on both parent and hybrid silicophosphate xerogels and provided a high number of Lewis acidic $\mathrm{Al}$ atoms on the surface of porous silicophosphate support (samples SiPL and SiC2SiPL, SA $=434$ and $458 \mathrm{~m}^{2} \mathrm{~g}^{-1}$, respectively). The high Lewis acidity was suggested on the basis of ${ }^{27} \mathrm{Al}$ MAS NMR spectra where only resonances of tetracoordinated $\mathrm{Al}$ atoms were observed (Fig. 6) and confirmed by pyridine adsorption. Samples after pyridine adsorption displayed an abrupt increase

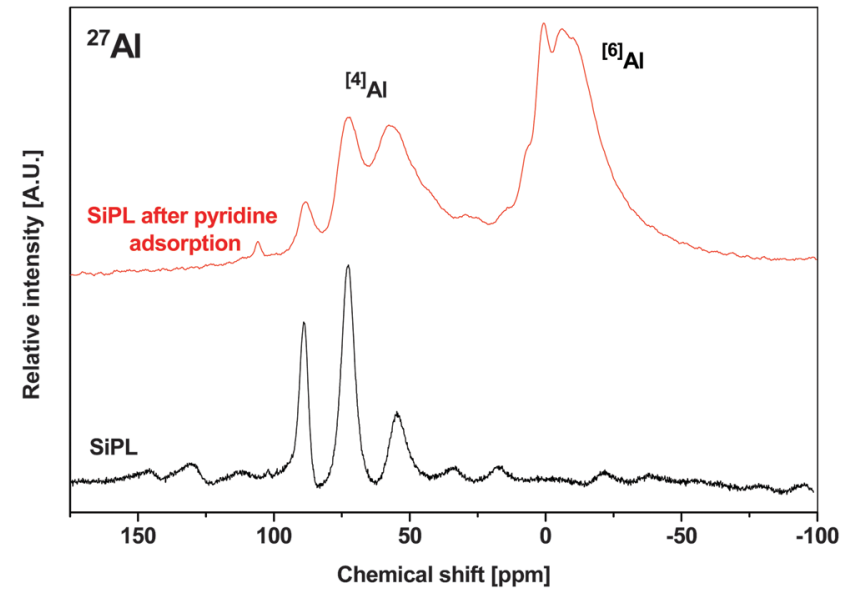

Fig. $6{ }^{27} \mathrm{Al}$ MAS NMR spectra of Lewis acidic xerogel (SiPL) before and after pyridine adsorption.

of the signal assigned to hexacoordinated aluminium atoms in ${ }^{27} \mathrm{Al}$ MAS NMR spectra (Fig. 6) and absorption bands at 1450, 1489 , and $1615 \mathrm{~cm}^{-1}$ in IR spectra typical for pyridine coordinated to a Lewis acid center (Fig. 5). These samples were tested in $\alpha$-methylstyrene dimerization and they displayed zero activity as expected. However this changed when moist methylstyrene was used for the catalytic tests. In this case hydrochloric acid was released in situ by the hydrolysis of residual $\equiv \mathrm{Al}-\mathrm{Cl}$ groups and catalyzed the dimerization similar to a published report where $\mathrm{AlCl}_{3}$ was tested. ${ }^{23}$

\section{Conclusions}

The surface reactions of silicophosphate xerogels with various reagents have been investigated and optimized to produce tailored Brønsted and Lewis acidic heterogeneous catalysts. The residual organic (acetoxy and trimethylsilyloxy) groups coming from the non-hydrolytic sol-gel synthesis were utilized in the reactions, however, the skeletal $\equiv \mathrm{Si}-\mathrm{O}-\mathrm{P} \equiv$ bonds have also been found to participate in these reactions. Water caused the elimination of acetoxy groups and hydrolysis of $\equiv \mathrm{Si-O}-$ $\mathrm{P} \equiv$ bonds which provided Brønsted acidic $\mathrm{HO}-\mathrm{P} \equiv$ groups. The rupture of skeletal bonds leads to an abrupt decrease of surface area. Hexamethyldisiloxane was effective in the removal of acetoxy groups from these matrices. $\mathrm{SiCl}_{4}$ and $\mathrm{POCl}_{3}$ reacted preferably with trimethylsilyloxy groups releasing trimethylchlorosilane and forming new $\equiv \mathrm{Si}-\mathrm{O}-\mathrm{P} \equiv$ and $\equiv \mathrm{P}-\mathrm{O}-\mathrm{P} \equiv$ bridges while acetoxy groups reacted less readily. The surface of silicophosphates was then covered by $\mathrm{Si}$ or $\mathrm{P}$ atoms with reactive residual chlorine atoms. Through stable $\mathrm{Si}-\mathrm{C}$ and $\mathrm{P}-\mathrm{C}$ bonds, various organic groups could be anchored to the surface adding new functionality to these xerogels. Finally, $\mathrm{AlMe}_{3}, \mathrm{Al}\left(\mathrm{NMe}_{2}\right)_{3}$, and $\mathrm{AlCl}_{3}$ were investigated as potential sources of tetrahedral aluminium atoms. The most promising results were obtained with aluminium trichloride which was not subject to ligand exchange and condensed smoothly with trimethylsilyloxy groups releasing trimethylchlorosilane and 
forming new $\equiv \mathrm{Al}-\mathrm{O}-\mathrm{P} \equiv$ bonds. The acetoxy groups coordinated readily to aluminium atoms in all cases and decreased their Lewis acidity.

The knowledge of studied reactions was utilized to synthesize Brønsted and Lewis acidic catalysts from silicophosphate xerogels (supports). The two-step synthesis $\left(\mathrm{POCl}_{3}, \mathrm{H}_{2} \mathrm{O}\right)$ provided porous solid Brønsted acids which showed high catalytic activities for the dimerization of $\alpha$-methylstyrene. The selectivity of the catalysts was dependent on their pore sizes. The second twostep protocol ( $\mathrm{HMDSO}, \mathrm{AlCl}_{3}$ ) resulted in the formation of porous and highly Lewis acidic xerogels. These are only two examples of procedures for tailoring the surface of silicophoshate xerogels and using them as potential supports for heterogeneous catalysts and other functional materials.

\section{Acknowledgements}

This research has been financially supported by the Ministry of Education, Youth and Sports of the Czech Republic under the project CEITEC 2020 (LQ1601).

\section{References}

1 P. G. Mingalyov and G. V Lisichkin, Russ. Chem. Rev., 2006, 75, 541-557.

2 J. H. Clark, D. J. Macquarrie and E. B. Mubofu, Green Chem., 2000, 2, 53-56.

3 J. A. Elings, R. Ait-Meddour, J. H. Clark and D. J. Macquarrie, Chem. Commun., 1998, 2707-2708.

4 X. Zhou, L. Zhong and Y. Xu, Inorg. Mater., 2008, 44, 976-979.

5 P. Tynjälä and T. T. Pakkanen, Microporous Mesoporous Mater., 1998, 20, 363-369.

6 P. M. Price, J. H. Clark, K. Martin, D. J. Macquarrie and T. W. Bastock, Org. Process Res. Dev., 1998, 2, 221-225.

7 J. H. Clark and D. J. Macquarrie, Chem. Commun., 1998, 853-860.

8 P. M. Price, J. H. Clark and D. J. Macquarrie, J. Chem. Soc., Dalton Trans., 2000, 101-110.

9 S. Pawsey, M. McCormick, S. De Paul, R. Graf, Y. S. Lee, L. Reven and H. W. Spiess, J. Am. Chem. Soc., 2003, 125, 4174-4184.

10 G. Guerrero, P. H. Mutin and A. Vioux, J. Mater. Chem., 2001, 11, 3161-3165.

11 C. Queffélec, M. Petit, P. Janvier, D. A. Knight and B. Bujoli, Chem. Rev., 2012, 112, 3777-3807.

12 R. Frantz, J.-O. Durand, M. Granier and G. F. Lanneau, Tetrahedron Lett., 2004, 45, 2935-2937.

13 C. Carbonneau, R. Frantz, J.-O. Durand, M. Granier, G. F. Lanneau and R. J. P. Corriu, J. Mater. Chem., 2002, 12, 540-545.
14 G. Guerrero, J. G. Alauzun, M. Granier, D. Laurencin and P. H. Mutin, Dalton Trans., 2013, 42, 12569.

15 E. L. Hanson, J. Schwartz, B. Nickel, N. Koch and M. F. Danisman, J. Am. Chem. Soc., 2003, 125, 16074-16080.

16 P. Thissen, A. Vega, T. Peixoto and Y. J. Chabal, Langmuir, 2012, 28, 17494-17505.

17 A. Vega, P. Thissen and Y. J. Chabal, Langmuir, 2012, 28, 8046-8051.

18 A. Styskalik, D. Skoda, Z. Moravec, J. G. Abbott, C. E. Barnes and J. Pinkas, Microporous Mesoporous Mater., 2014, 197, 204-212.

19 A. Styskalik, D. Skoda, Z. Moravec, M. Babiak, C. E. Barnes and J. Pinkas, J. Mater. Chem. A, 2015, 3, 7477-7487.

20 J. H. Balthis, E. G. Rochow and D. G. White, in Inorganic Syntheses, ed. J. C. Bailar, Jr., John Wiley \& Sons Inc., 2007, pp. 45-47.

21 J. Rouquerol, F. Rouquerol and K. S. W. Sing, Adsorption by Powders and Porous Solids, Academic Press, 1998.

22 S. Lowell, Characterization of Porous Solids and Powders: Surface Area, Pore Size and Density, Springer, 2004.

23 G. N. Kirichenko, N. G. Grigor'eva, V. I. Glazunova and U. M. Dzhemilev, Pet. Chem., 2009, 49, 306-310.

24 Y. Song, Y. Huang, E. A. Havenga and I. S. Butler, Vib. Spectrosc., 2001, 27, 127-134.

25 J. Livage, P. Barboux, M. T. Vandenborre, C. Schmutz and F. Taulelle, J. Non-Cryst. Solids, 1992, 147-148, 18-23.

26 S. Jähnigen, E. Brendler, U. Böhme, G. Heide and E. Kroke, New J. Chem., 2014, 38, 744.

27 C. Coelho, T. Azais, C. Bonhomme, L. Bonhomme-Coury, C. Boissière, G. Laurent and D. Massiot, C. R. Chim., 2008, 11, 387-397.

28 S. Kvisle and E. Rytter, J. Mol. Struct., 1984, 117, 51-57.

29 A. Vioux, Chem. Mater., 1997, 9, 2292-2299.

30 W. Uhlig and C. Tretner, J. Organomet. Chem., 1994, 467, 31-35.

31 J. H. Wengrovius, M. F. Garbauskas, E. A. Williams, R. C. Goint, P. E. Donahue and J. F. Smith, J. Am. Chem. Soc., 1986, 108, 982-989.

32 E. Kupce and E. Lukevics, J. Magn. Reson., 1988, 76, 63-73.

33 R. J. P. Corriu and D. Leclercq, Angew. Chem., Int. Ed., 1996, 35, 1420-1436.

34 M. Toba, F. Mizukami, S. Niwa, T. Sano, K. Maeda and H. Shoji, J. Mater. Chem., 1994, 4, 1131-1135.

35 M. Nara, H. Torii and M. Tasumi, J. Phys. Chem., 1996, 100, 19812-19817.

36 G. B. Deacon and R. J. Phillips, Coord. Chem. Rev., 1980, 33, 227-250.

37 A. Styskalik, D. Skoda, Z. Moravec, P. Roupcova, C. E. Barnes and J. Pinkas, RSC Adv., 2015, 5, 73670-73676. 